\title{
Rapid-Scan, Polarimetric, Doppler Radar Observations of Tornadogenesis and Tornado Dissipation in a Tornadic Supercell: The "El Reno, Oklahoma" Storm of 24 May 2011*
}

\author{
JANA LESAK HOUSER \\ Department of Geography, Ohio University, Athens, Ohio \\ HowARd B. BLuestein AND JEFFREY C. SNYDER \\ School of Meteorology, University of Oklahoma, Norman, Oklahoma
}

(Manuscript received 1 August 2014, in final form 12 February 2015)

\begin{abstract}
On 24 May 2011, a mobile, rapid-scan, X-band, polarimetric, Doppler radar (RaXPol) collected data on a supercell as it produced two tornadoes near El Reno, Oklahoma. The first tornado, rated an EF-3, was documented from intensification to decay, and the genesis and intensification of a second tornado that was rated an EF-5 was subsequently also documented.

The objective of this study is to examine the spatiotemporal evolution of the rotation associated with the tornadoes (i) as the first tornado weakened to subtornadic intensity and (ii) as the second tornado formed and intensified. It is found that weakening did not occur monotonically. The transition from tornadic to subtornadic intensity over the depth of the radar volume $(\sim 4 \mathrm{~km})$ occurred in less than $30 \mathrm{~s}$, but this behavior is contingent upon the threshold for Doppler shear used to define the tornado. Similarly, the onset of a tornadicstrength Doppler velocity couplet occurred within a 30-s period over all elevations.

Additionally, the evolution of storm-scale features associated with tornado dissipation and tornadogenesis is detailed. These features evolved considerably over relatively short time intervals (1-4 min). It is shown that during the transition period between the two tornadoes, two mesocyclones were present, but neither the tornadoes nor the mesocyclones evolved in a manner entirely consistent with any published conceptual model of supercell cycling, although certain aspects were similar to classic conceptual models. The mesocyclone and the tornado evolved differently from each other, in a manner that resembles a hybrid between the occluding and nonoccluding cyclic mesocyclogenesis models presented by Adlerman and Droegemeier.
\end{abstract}

\section{Introduction}

Despite a growing body of literature describing observations of tornadogenesis and dissipation (Roberts and Wilson 1995; Wakimoto and Atkins 1996; Dowell and Bluestein 1997, 2002b; Wakimoto and Liu 1998; Dunn and Vasiloff 2001; Ziegler et al. 2001; Bluestein and Wakimoto 2003; Bluestein et al. 2003; Wakimoto et al. 2004; Van Den Broeke et al. 2008; Wurman et al.

\footnotetext{
* Supplemental information related to this paper is available at the Journals Online website: http://dx.doi.org/10.1175/MWR-D14-00253.s1.

Corresponding author address: Jana Houser, Ohio University, 145 Clippinger Laboratories, Athens, OH 45701.

E-mail: houserj@ohio.edu
}

2007, 2010; Markowski and Richardson 2009; Palmer et al. 2011; Marquis et al. 2012; Tanamachi et al. 2012; Kosiba et al. 2013; Kosiba and Wurman 2013; French et al. 2013, hereafter F13; French et al. 2014, hereafter F14; Davies-Jones 2015), these processes still are not completely understood. Even though a number of studies have successfully addressed the kinematics and dynamics of these processes, many of the details surrounding tornado formation and decay remain unclear.

A nontrivial challenge that has thus far precluded a more complete understanding of tornado-scale processes is the short time scale over which many tornadoes form and evolve. For a "typical" 100-m-wide tornado, the advective time scale is $\sim 10 \mathrm{~s}$ (Bluestein et al. 2003, 2010). Conventional observational methods miss the details of such processes partly owing to inadequate temporal resolution. Recently, mobile, rapid-scanning 
radars have been utilized in the field to obtain data with improved temporal resolution (Wurman and Randall 2001; Bluestein et al. 2010; Pazmany et al. 2013; F13; Kosiba and Wurman 2013; Kosiba et al. 2013; F14; Snyder and Bluestein 2014). These studies have demonstrated the success of rapid-scan platforms, but only F13 and F14 have examined the details of tornadogenesis and/or tornado dissipation using such a platform. Kosiba and Wurman (2013) document a portion of the decay process with the Rapid-Scan Doppler on Wheels (DOW), but data collection terminated before the tornado completely dissipated. Similarly, there are only a small number of cases for which both temporal and spatial resolution are adequate to resolve tornado-scale processes (Wurman 2002; Wurman et al. 2007; Bluestein et al. 2010; Wakimoto et al. 2011; Kosiba and Wurman 2013; Wurman and Kosiba 2013; Snyder and Bluestein 2014), and none of these analyze tornadogenesis or decay in detail.

One common notion used to explain tornadogenesis is that tornadoes produced by mesocyclones form aloft initially and then build downward with time in a manner consistent with the dynamic pipe effect (DPE; Leslie 1971). Many studies conducted prior to this decade, both observational (e.g., Burgess et al. 1975; Brown et al. 1978; Trapp and Davies-Jones 1997; Trapp et al. 1999) and numerical (e.g., Leslie 1971; Smith and Leslie 1979; Wicker and Wilhelmson 1995), have supported this hypothesis. However, other studies (Vasiloff 1993; Trapp and Fiedler 1995; Trapp and Davies-Jones 1997; Alexander 2010; F13; F14) have observed rotation developing initially near the surface and either building upward with time or nearly simultaneously intensifying over a relatively deep layer of the lower troposphere during tornadogenesis. Unfortunately, these studies have lacked the temporal resolution to determine clearly the evolution of rotation. F13 examined three cases of tornadogenesis using a mobile phased array radar [i.e., the Mobile Weather Radar 2005 X-band Phased array (MWR-05XP); Bluestein et al. (2010)] capable of sector volumetric updates of 6-14 s and did not find evidence of a descending tornado vortex signature (TVS) in any of the cases. Unfortunately, the phased array engineering that enabled rapid temporal updates with this radar also compromised the angular resolution of the instrument; the half-power beamwidth in the azimuthal direction is $1.8^{\circ}$ and is $2.0^{\circ}$ in the vertical (although oversampling is performed every $1.5^{\circ}$ ). This corresponds to an azimuthal resolution of $\sim 160 \mathrm{~m}$ in the best case study of F13 and F14, and more typically to an azimuthal resolution $>400 \mathrm{~m}$. Thus, while the datasets analyzed by F13 and F14 present a unique opportunity to examine the volumetric evolution of rotation associated with tornadoes over short time intervals, it is usually not possible to resolve the actual tornado well, if at all.
TABLE 1. Summary of tornadoes observed by RaXPol used for this case study on 24 May 2011.

\begin{tabular}{|c|c|}
\hline Parameter & Value \\
\hline \multicolumn{2}{|l|}{ Tornado No. 1} \\
\hline NWS EF rating (as of June 2014) & EF-3 \\
\hline $\begin{array}{l}\text { Estimated peak winds } \\
\quad \text { (ground survey) }\end{array}$ & $140 \mathrm{mph}\left(62.5 \mathrm{~m} \mathrm{~s}^{-1}\right)$ \\
\hline Start time & 2031 UTC \\
\hline Start location & $\begin{array}{l}4 \mathrm{mi}(6.4 \mathrm{~km}) \mathrm{WSW} \\
\text { Lookeba, OK }\end{array}$ \\
\hline End time & 2047 UTC \\
\hline End location & 4 mi NE Lookeba, OK \\
\hline Damage pathlength & $9 \mathrm{mi}(14.5 \mathrm{~km})$ \\
\hline \multicolumn{2}{|l|}{ Tornado No. 2} \\
\hline NWS EF rating (as of June 2014) & EF-5 \\
\hline $\begin{array}{l}\text { Estimated peak winds } \\
\quad \text { (ground survey) }\end{array}$ & $>210 \mathrm{mph}\left(93.9 \mathrm{~m} \mathrm{~s}^{-1}\right)$ \\
\hline Start time & 2051 UTC \\
\hline Start location & 4 mi ESE Hinton, OK \\
\hline End time & 2135 UTC \\
\hline End location & 4 mi NE Guthrie, OK \\
\hline Damage pathlength & $65 \mathrm{mi}(104.6 \mathrm{~km})$ \\
\hline
\end{tabular}

This study is based on data collected by a mobile, rapid-scan, X-band, polarimetric radar (RaXPol; Pazmany et al. 2013) to examine the rapidly evolving processes associated with two tornadoes that occurred on 24 May 2011, southwest of El Reno, Oklahoma (Table 1). The dataset focuses on the maturation and decay of an (enhanced Fujita) EF-3 tornado and the subsequent genesis, and intensification of a second tornado that was rated EF-5. ${ }^{1}$ The combination of polarimetric and fine spatiotemporal resolution observations of the evolution of one strong tornado and one violent tornado, including genesis of the violent tornado, makes this study unique. ${ }^{2}$ This study addresses the following questions: 1) What is the spatiotemporal evolution of the rotation ${ }^{3}$ associated with the tornadoes as they develop, intensify, and decay? 2) During tornadogenesis, is there evidence that rotation develops aloft and builds downward, develops near the surface and builds upward,

\footnotetext{
${ }^{1}$ Additional data were collected before and after these phases, but are not discussed in the context of this study.

${ }^{2}$ An antenna rotation rate of $180^{\circ} \mathrm{s}^{-1}$ over $360^{\circ}$ PPIs at nine elevation angles yielded a volume acquisition time of $\sim 17 \mathrm{~s}$. With ranges between 16 and $8 \mathrm{~km}$ (dissipation and genesis, respectively), a $1^{\circ}$ beamwidth implies angular resolution of $\sim 280-140 \mathrm{~m}$. However, the effective horizontal beamwidth due to beam smearing associated with the rapid rotation rate is $1.5^{\circ}$, which degrades the effective resolution to $\sim 420-210 \mathrm{~m}$, respectively.

${ }^{3}$ The term "rotation" is used when referring to the strength of the tornado according to the Doppler velocity couplet. The term "vorticity" is not used because the two-dimensional wind components are not available.
} 
or neither? 3) How are storm-scale features related to the formation, intensification, and decay of the tornadoes? Although the questions posed here are not new, they either remain largely unanswered or incompletely explained. A brief discussion of the instrumentation and methodology used for this study is given in section 2. Results are presented in section 3, followed by a discussion in section 4 . Finally, conclusions are summarized in section 5 .

\section{Instrumentation, dataset, and methodology}

The need for rapid temporal resolution ${ }^{4}$ while maintaining high spatial resolution motivated the design for the instrument used in the current study. RaXPol was designed with the primary purpose of studying rapidly evolving convective phenomena (Pazmany et al. 2013).

The radar is equipped with a $2.4-\mathrm{m}$ diameter parabolic dish capable of transmitting and receiving both horizontally and vertically polarized waves. Polarimetric signatures enhance our ability to locate various stormscale features including updrafts (e.g., Kumjian and Ryzhkov 2008; Snyder et al. 2013). Specifications for the radar are summarized in Table 2 . The antenna mechanically rotates azimuthally at a maximum rate of just over $180^{\circ} \mathrm{s}^{-1}$. For much of this study, volume scans containing the full $360^{\circ}$ azimuthal circle and 9 elevation angles were completed in $\sim 17 \mathrm{~s}$; such a rapid scanning rate is enabled by a high-speed pedestal. To obtain enough independent samples to calculate accurate estimates for the retrieved radar variables, a frequency diversity technique-frequency hopping (Doviak and Zrnić 2006, p. 180)—is employed. Frequency hopping speeds the convergence of retrieved variable estimates

\footnotetext{
${ }^{4}$ The term "rapid" is used in the context of this paper to refer to volumetric radar updates and storm features that exhibit considerable morphology on time scales that are less than the time it takes for traditional mobile radars to sample a complete radar volume ( $\sim 2 \mathrm{~min}$ ). Volumetric updates for the RaXPol are consistently less than $20 \mathrm{~s}$ thus implying "rapid scanning." The orbital advective time scale $T$ of a tornado is typically $10-20$ s (i.e., much shorter than the 2-min traditional mobile radar volume scanning time) according to the definition: $T=2 \pi L / V$, where $L$ is a length scale and $V$ is a velocity scale. For a 100 -m-wide tornado with winds of $30 \mathrm{~m} \mathrm{~s}^{-1}$, $T \approx 21 \mathrm{~s}$ and the tornado is therefore considered a "rapidly evolving feature." Some of the storm-scale features (precipitation surges, RFGF surges, cycling mesocyclones) evolved over slightly longer periods, with advective time scales being $\sim 1-4 \mathrm{~min}$ based upon a linear advective time scale $(T=L / V)$, the larger size of the features $[O(L)=$ several kilometers], and the velocities they were associated with $\left[O(V)=10-20 \mathrm{~m} \mathrm{~s}^{-1}\right]$. These time scales are still shorter than the update time of WSR-88Ds ( $\sim 4.5 \mathrm{~min})$; and, only 1-2 samples could be acquired by traditional mobile radars in this time. Thus, we consider these features to "evolve rapidly" as well.
}

TABLE 2. Instrument specifications for RaXPol for this dataset. [Adapted from Pazmany et al. (2013).]

\begin{tabular}{ll}
\hline \multicolumn{1}{c}{ Parameter } & \multicolumn{1}{c}{ Value } \\
\hline Center frequency & $9.73 \mathrm{GHz} \pm 20 \mathrm{MHz}$ \\
Transmit power & $20 \mathrm{~kW}$ peak, 200 W avg \\
Transmit pulse width & $0.1-40 \mu \mathrm{s}$ \\
Transmit polarization & Equal power V/H \\
PRF & Uniform or staggered \\
Antenna type & Dual-linear polarized parabolic \\
& $\quad$ reflector \\
Antenna diameter & $2.4 \mathrm{~m}$ \\
Half-power beamwidth & $1.0^{\circ}$ \\
Half-power antenna gain & $44.5 \mathrm{~dB}$ \\
Maximum pedestal scan & $180^{\circ} \mathrm{s}^{-1}$ azimuthal; 36 ${ }^{\circ}{ }^{-1}$ elevation \\
$\quad$ rate & \\
Receiver noise figure & $3 \mathrm{~dB}$ \\
Receiver bandwidth & $0.5-40 \mathrm{MHz}$, or custom \\
Range resolution & $150 \mathrm{~m}$ until 2052 UTC, 75 m afterward \\
Range gate spacing & $75 \mathrm{~m}$ until 2052 UTC, $15 \mathrm{~m}$ afterward \\
\hline
\end{tabular}

to the mean compared to conventional single-frequency transmission methods by providing more quasiindependent samples more quickly. For a complete description of RaXPol's specifications and technology, the reader is referred to Pazmany et al. (2013).

The biggest limitation of the data utilized in this study is that the lowest elevation angle for which volumetric data are available is only $4^{\circ}$, which is relatively high. The scanning strategy employed began at $2^{\circ}$, increasing by increments of $2^{\circ}$ until an elevation angle of $18^{\circ}$. However, an unforeseen consequence of the rapid scanning rate (this was the first year of data collection with this instrument) was that the antenna transition from $18^{\circ}$ to $2^{\circ}$ elevation occurred within the $360^{\circ} \mathrm{PPI}$ of the $2^{\circ}$ elevation angle, effectively causing the data recorded within the $2^{\circ}$ scan to be a downward spiral from $18^{\circ}$. This problem caused the antenna to be positioned physically at $2^{\circ}$ only over a narrow azimuthal sector of $\sim 30^{\circ}$. A minimum elevation angle of $4^{\circ}$ translates into a lowest vertical height above radar level (ARL) of about $1 \mathrm{~km}$ during tornado decay and about $650 \mathrm{~m}$ during tornadogenesis. We recognize the limitation this places on examining low-level processes during the dissipation and genesis periods. However, this shortcoming does not impact the determination of whether the tornadic rotation developed aloft or at low levels initially. If tornadic rotation formed aloft first and progressively descended to lower levels with time, or if the rotation developed near the surface and strengthened upward with time, these trends would be visible in the data available regardless of the whether the lowest available height were $1 \mathrm{~km}$ or $100 \mathrm{~m}$.

RaXPol was deployed at $35.5077^{\circ} \mathrm{N}, 98.2346^{\circ} \mathrm{W}$, $\sim 2.8 \mathrm{~km}$ south-southeast (SSE) of exit 108 on I-40 (Fig. 1). Rapid-scan data collection began at $\sim 2036$ UTC 




FIG. 1. Tornado tracks and location of the RaXPol (black star). (Courtesy of J. Snyder.)

and ended at $\sim 2116$ UTC; this study will focus on events that occurred between $\sim 2040$ and 2055 UTC. The majority of data used in this study comes directly from single-Doppler observations. The intensity of rotation was quantified by the traditional proxy variable $\Delta V_{\max }$ representing the difference between the maximum outbound and maximum inbound radial velocity measurements.

Additionally, velocity couplets were only considered when $\Delta V_{\max }$ was $>20 \mathrm{~m} \mathrm{~s}^{-1}$, and the distance between $V_{\text {maxinbound }}$ and $V_{\text {maxoutbound }}$ was $<2 \mathrm{~km}$, implying that the vorticity associated with velocity couplets must exceed $2 \times 10^{-2} \mathrm{~s}^{-1}$. The values for $V_{\max }$ were determined manually by finding the pixel in the raw radar data that had the most positive outbound or most negative inbound radial velocity value for all times when a velocity couplet was qualitatively discernable, despite whether or not a tornado was observed. This decision was made for several reasons. First, information about the tendencies of subtornadic rotation was desired to provide insight into the pretornadic and posttornadic evolution of rotation. Additionally, the $\Delta V_{\max }$ value that best corresponded with the start and end times of the tornado according to the NWS survey ${ }^{5}$ was $55 \mathrm{~m} \mathrm{~s}^{-1}$, which is

\footnotetext{
${ }^{5}$ It is possible that a tornado can be on the ground without being assessed by a damage survey if damage indicators are not present, as is sometimes the case in open prairie. However, owing to the prevalence of damage indicators in the vicinity where tornadogenesis occurred (in a wooded river valley), the space-time correlation between the location of NWS damage indicators and the radar observations of the velocity couplet location, and the timing of visual observations of debris made by the radar crew, the authors are reasonably confident that the damage survey start time was accurate to within $\sim 30 \mathrm{~s}$.
}

higher than that of many other studies (Trapp et al. 1999; Alexander 2010; Marquis et al. 2012; F13; Kosiba et al. 2013). The higher threshold is likely due to a combination of the relatively higher spatial resolution when compared with F13 and the availability of a high-quality damage assessment to reduce the uncertainty regarding tornado positions or locations at the time of genesis. Additionally, depending on the vertical profile of rotation, the relatively high beam height of $\sim 650 \mathrm{~m}$ ARL may be a factor. If rotation were to increase with height above $\sim 100 \mathrm{~m}$, then the tornadic wind speeds at levels where many other mobile radars typically acquire observations would be below $55 \mathrm{~m} \mathrm{~s}^{-1}$.

The volumetric data were objectively analyzed using a Barnes's scheme and reconstructed into a threedimensional, Cartesian grid to provide smoother representation of the data in the vertical by interpolating between observed elevations angles. A second method of examining the rotation of the tornado and its surroundings was employed by calculating a pseudovorticity quantity $\left(\zeta_{\mathrm{ps}}\right)$ from the $3 \mathrm{D}$ objectively analyzed grids. The range from the radar to each point on the analysis grid was calculated, and a series of nearly constant radius arcs was created for each grid point. The grid was kept in Cartesian coordinates, and the two adjacent grid points that most closely represented a constant radius were used to construct the arcs. Pseudovorticity was calculated as

$$
\zeta_{\mathrm{ps}}=\frac{2 \Delta V_{r}}{\Delta r}
$$

where $\Delta V_{r}$ is the change in observed radial velocity to either side of a specified grid point along the constant radius arcs, and $\Delta r$ is the azimuthal distance between the grid points for which $\Delta V_{r}$ is calculated. 


\section{Observations and discussion}

Rapid-scan data acquisition began at 2036:30 UTC, approximately $5.5 \mathrm{~min}$ after the genesis of tornado 1 (not visible to the radar crew) (NWS damage report, tornado B1; NWS 2011a). At the time data collection began, tornado 1 was located at a range of $\sim 23.5 \mathrm{~km}$ from the radar. Data acquisition ceased at $\sim 2116$ UTC at which time a second, violent tornado was moving away from the radar crew (NWS damage report, tornado B2; NWS 2011b). This study uses data collected prior to the demise of the first tornado (beginning around 2040 UTC) through the intensification of the second tornado, ending around 2055 UTC. The scanning strategy after 2055 UTC was much different; the later evolution of the tornado will be addressed in a subsequent paper.

\section{a. Tornado decay}

\section{1) StORM-SCALE EVOLUTiON}

\section{(i) Midlevels}

Storm-scale processes must be examined prior to the onset of decay (which occurred at approximately 2044 UTC) in order to better understand the tornado dissipation process. Changes in the storm structure prior to decay appeared to begin at midlevels. Between 2040 and 2042 UTC, the mesocyclone between $\sim 3$ and $5 \mathrm{~km}$ above radar level (ARL) (hereafter all other heights denote elevations ARL unless otherwise specified) broadened and weakened slightly, having two regions of quasi-concentric vorticity maxima: an inner one with a diameter of $\sim 6 \mathrm{~km}$ and an outer one with a diameter of $\sim 12 \mathrm{~km}$. During this time, a partial ${ }^{6} Z_{\mathrm{DR}}$ ring was evident, outlining the eastern periphery of the mesocyclone and, presumably, the updraft (e.g., Kumjian and Ryzhkov 2008; Snyder et al. 2013) (Fig. 2). Between 2042 and 2044 UTC, a second partial $Z_{\mathrm{DR}}$ ring appeared to the SE of the original one, and a new midlevel mesocyclone organized to the east-southeast (ESE) of the original one. The new mesocyclone was not independent from the first one. It developed as the distance between the concentric inbound (outbound) velocities widened (closed), splitting the original inbound velocities

\footnotetext{
${ }^{6}$ It is possible that the $Z_{\mathrm{DR}}$ ring was more complete, but attenuation at the rear of the storm diminished what may have been the rear half of the ring. Methods exist to estimate attenuation and differential attenuation at X band (e.g., Snyder et al. 2010), although the vast majority of previous work has focused on attenuation through rain. The effects of attenuation are not estimated in this paper since anomalous attenuation through mixed-phase precipitation within the updraft above the freezing level introduces significant uncertainties in the relevant parameters that must be specified a priori when conventional correction methods are used.
}

into two distinct regions of cyclonic shear (Fig. 2, $V_{r}$ column); and it appeared to be shed from the parent mesocyclone in a manner similar to that described by Dowell and Bluestein (2002a, hereafter DB02a), The midlevel tornado signature (TS) $)^{7}$ (white circle, Fig. 2) propagated to the ESE during this process, becoming tethered to the new mesocyclone rather than remaining attached to the original. The midlevel TS continued to propagate nearly due east while storm motion was to the northeast, displacing it farther from the original mesocyclone (see online supplemental material for an animation of this process). Several minutes later, the tornadic circulation became displaced from the maximum cyclonic shear associated with new mesocyclone, and shortly afterward, dissipated (Fig. 3).

\section{(ii) Low levels}

Throughout the mature phase of tornado 1 , low-level $\left(4^{\circ}\right.$ elevation angle, $\sim 1.2 \mathrm{~km}$ ) storm-relative outflow to the southwest of the tornado [associated with the rear-flank downdraft, (RFD)] was adjacent to a region of relatively strong storm-relative inflow, $>40 \mathrm{~ms}^{-1}$ (Fig. 4a). The outflow boundary, manifest by a band of near-zero $V_{r}$, remained quasi-stationary in a storm-relative sense, suggesting a balance may have been achieved between the rear-flank downdraft outflow and the storm inflow during this time. Between 2041 and 2046 UTC, the inflow weakened and the outflow behind the rear-flank gust front (RFGF) increased, causing the RFGF to surge eastward (Fig. 4). Simultaneously, a decrease in copolar correlation coefficient $\left(\rho_{\mathrm{hv}}\right)$ (suggesting an increased concentration of nonmeteorological scatterers within the radar volume likely due to convergence) and the advancement of precipitation through the hook echo leading to a "hammerhead" reflectivity (Bluestein et al. 2014) appearance were observed (Fig. 4, left column). This surge in precipitation did not appear to be associated with a descending reflectivity core (Rasmussen et al. 2006; Byko et al. 2009). Rather, heavy precipitation originating on the rear side of the forward flank was wrapped around the western periphery of the mesocyclone. Thus, this surge in precipitation and outflow does not appear to have had its origin in the RFD, but rather in the forward-flank downdraft (FFD).

During the last several minutes of tornado 1's life, the low-level TS became displaced from the tip of the hook and propagated toward the southeastern flank of the hook, toward the apex of the hammerhead feature

\footnotetext{
7 "Tornado signature" is used here and throughout the document when the resolution is high enough that the core region is comparable in size to the $1^{\circ}$ azimuthal beamwidth at the range of the tornado ( $\sim 330 \mathrm{~m}$ here) (Brown and Wood 2012). When resolution is not sufficient to resolve the core radius, the term "tornado vortex signature" is used.
} 

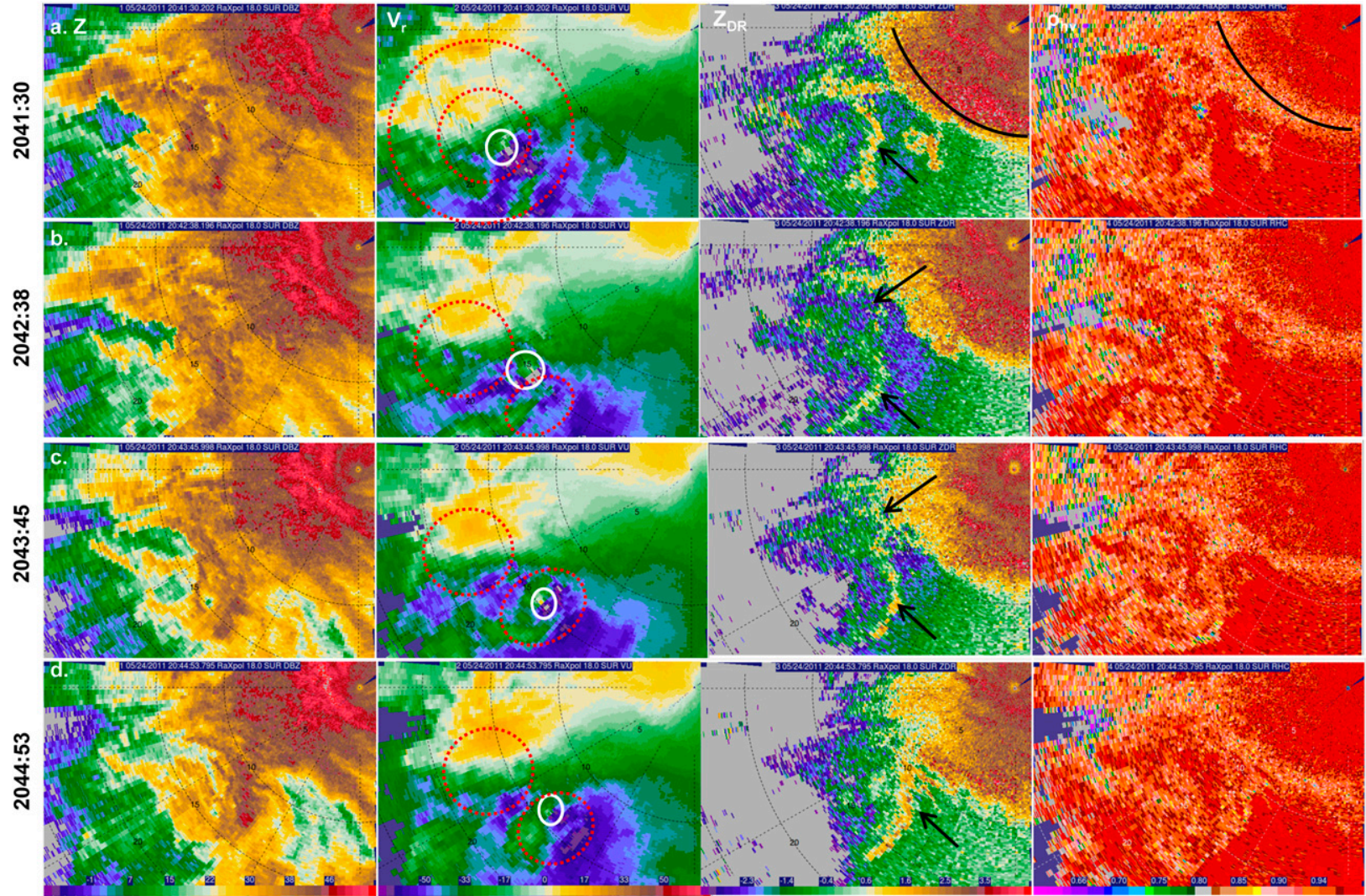

FIG. 2. (from left to right) Reflectivity factor $(\mathrm{dBZ})$, radial velocity $\left(\mathrm{m} \mathrm{s}^{-1}\right)$, differential reflectivity $(\mathrm{dB})$, and $\rho_{\mathrm{hv}}$ every $\sim 68 \mathrm{~s}$ at $18^{\circ}$ elevation angle at (a) 2041:30, (b) 2042:38, (c) 2043:45, (d) 2044:53, (e) 2046:00, (f) 2047:08, (g) 2048:15, and (h) 2049:21 UTC of the cycling mesocyclone (red dashed circles), TS of tornado 1 (white circles), partial $Z_{\mathrm{DR}}$ rings/column (black arrows), and BWER (red arrows). Black curves indicate the approximate melting level.

(Fig. 4, left two columns; see online supplemental material for an animation of this process). This process occurred at the same time as the transition of the midlevel TS from the original mesocyclone to the new one. From the $\rho_{\text {hv }}$ field, the RFGF appears to wrap into the tornadic vortex region between 2045:30 and 2046:37 UTC (Figs. 4c,d, right column), implying an occlusion of the low-level mesocyclone by outflow around the tornado. The occlusion remained confined to the northern tip of the hook, and the outflow boundary never completely cut off the updraft from the environmental inflow. By 2047:00 UTC the tornado had dissipated. Although the tornado was no longer causing surface damage, the remnant subtornadic circulation remained intact for several minutes after the tornado dissipated.

\section{2) TornAdo-SCAlE EVOlution}

To address how the rotation associated with the tornado evolved, a sequence of time-height analyses was made by documenting $\Delta V_{\max }$ for every elevation angle over time (Fig. 5). Tornado 1's $\Delta V_{\max }$ reached its peak intensity at all elevation angles between 2042 and 2044 UTC and subsequently weakened over the next 3 min until the tornado dissipated. The onset of tornado decay occurred at $\sim$ 2044:00 UTC when $\Delta V_{\max }$ decreased consistently over all elevation angles (Fig. 5b). Initially, $\Delta V_{\max }$ decreased rapidly and nearly simultaneously $\left(\sim 40 \mathrm{~m} \mathrm{~s}^{-1}\right.$ over $\left.\sim 50 \mathrm{~s}\right)$ over the depth for which data were available (1-5 km at this time). After this initial rapid weakening, further weakening did not occur simultaneously at all elevations, nor was it monotonic with time. At elevation angles above $\sim 3 \mathrm{~km}$, there was a brief period of reintensification suggesting the process or processes driving tornado decay were not constant, or that intensification processes were superimposed upon decay processes. The velocities initially weakened most quickly between about 1 and $3 \mathrm{~km}$ but persisted at weak tornadic intensities afterward. The magnitude of $\Delta V_{\max }$ at all heights became subtornadic nearly simultaneously ${ }^{8}$ as velocities

\footnotetext{
${ }^{8}$ The minimum beam height at the time of tornado decay was $>800 \mathrm{~m}$. Therefore, the behavior of $\Delta V_{\max }$ near the ground during tornado decay could be different from what was observed by the radar. The maximum beam height was $\sim 4.25 \mathrm{~km}$.
} 


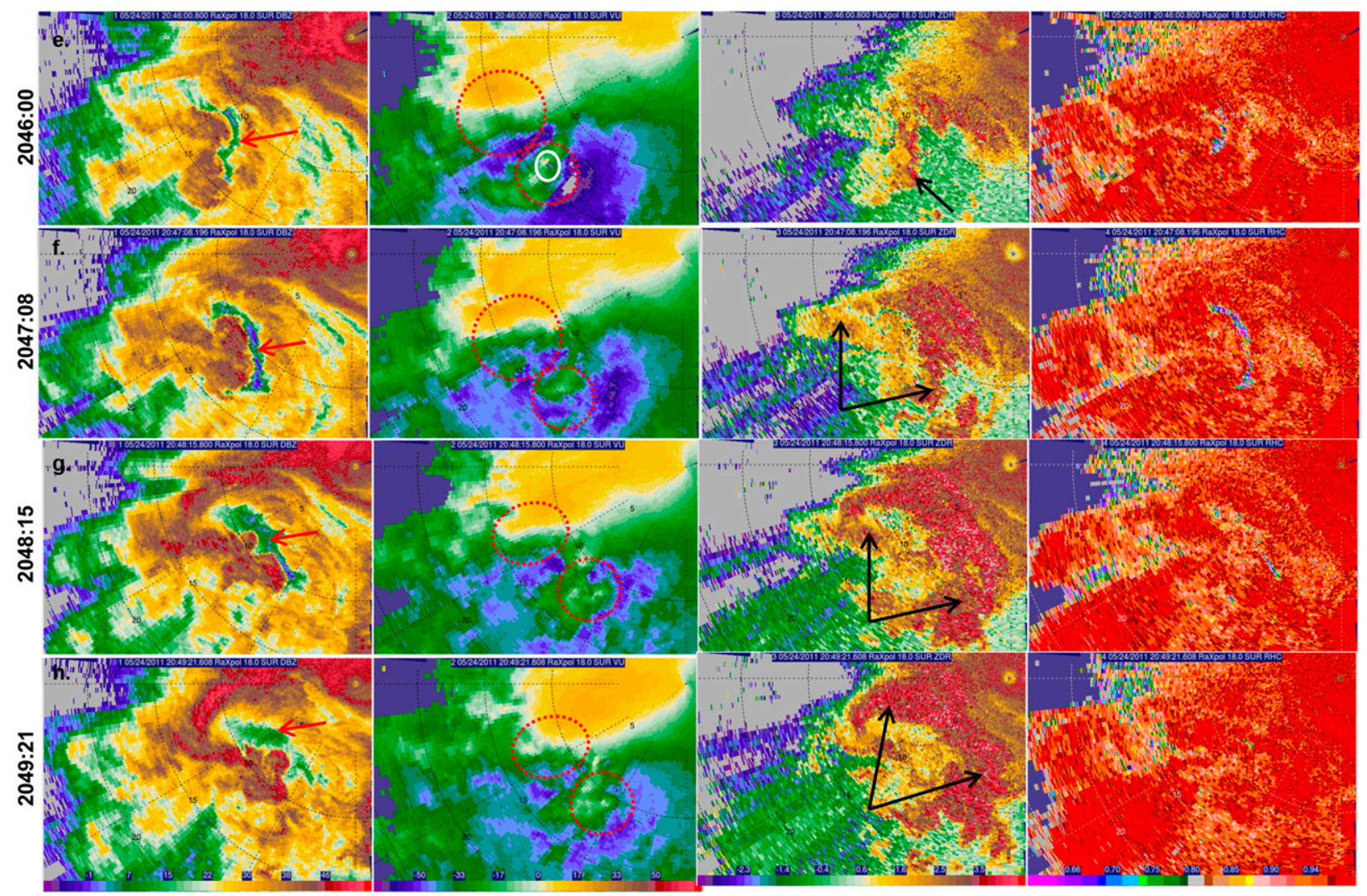

FIG. 2. (Continued)

were reduced below the tornadic threshold within two consecutive volume scans, or $\sim 30$ s of each other over the depth of the observed domain. According to the NWS damage survey (NWS 2011c), the tornado officially dissipated around 2047:00 UTC, which matches well with the time that $\Delta V_{\max }$ decreased below $55 \mathrm{~m} \mathrm{~s}^{-1}$ (Fig. 5b).

Another way of examining the trends of rotation and tornado structure is to examine $\zeta_{\mathrm{ps}}$ three dimensionally, using objectively analyzed data. Using $\zeta_{\text {ps }}$ enables a more spatially comprehensive analysis of rotation. In addition, structural characteristics such as the size and the tilt of the vortex can easily be discerned. A sequence of $\zeta_{\mathrm{ps}}$ (bound by the $0.15 \mathrm{~s}^{-1}$ contour) associated with the decay of tornado 1 is presented in Fig. 6. It is seen that the evolution of $\zeta_{\mathrm{ps}}$ is consistent with that of $\Delta V_{\max }$, implying that the strengthening and weakening trends observed in the $\Delta V_{\max }$ analyses are applicable to the larger-scale tornado vortex and not just the velocity couplet. It is also seen that tornado 1 clearly began to weaken first between 2043:38 and 2044:12 UTC around $z \sim 2-2.5 \mathrm{~km}$. It briefly reintensified at most levels at 2046 UTC, and then rapidly weakened again at all heights within the domain between 2046:26 and 2046:43 UTC.
Just prior to the final decay, it again weakened first between 1.5 and $2.5 \mathrm{~km}$. The lowest elevation angles retained the strongest and most spatially extensive rotation longer than the higher elevations (2046:43 UTC in Fig. 6).

\section{b. Genesis and intensification of tornado 2}

\section{1) STORM-SCALE REORGANIZATION PRIOR TO AND DURING TORNADOGENESIS \\ (i) Midlevels}

At midlevels $(z \sim 3-4 \mathrm{~km})$ prior to tornadogenesis ( 2048 UTC), the two mesocyclones previously mentioned in section 3 were still present. The western mesocyclone was broad and quasi symmetric but the circulation with the eastern (new) one remained relatively weak and disorganized. Multiple small, transient vortices were evident in the eastern one (Fig. 7). Over the next several minutes ( 2045-2049 UTC), the inbound velocities of the western mesocyclone weakened and the wind field began to reorganize into a single, broad area of rotation (not shown). The mesocyclone at this height remained broad and disorganized until the 

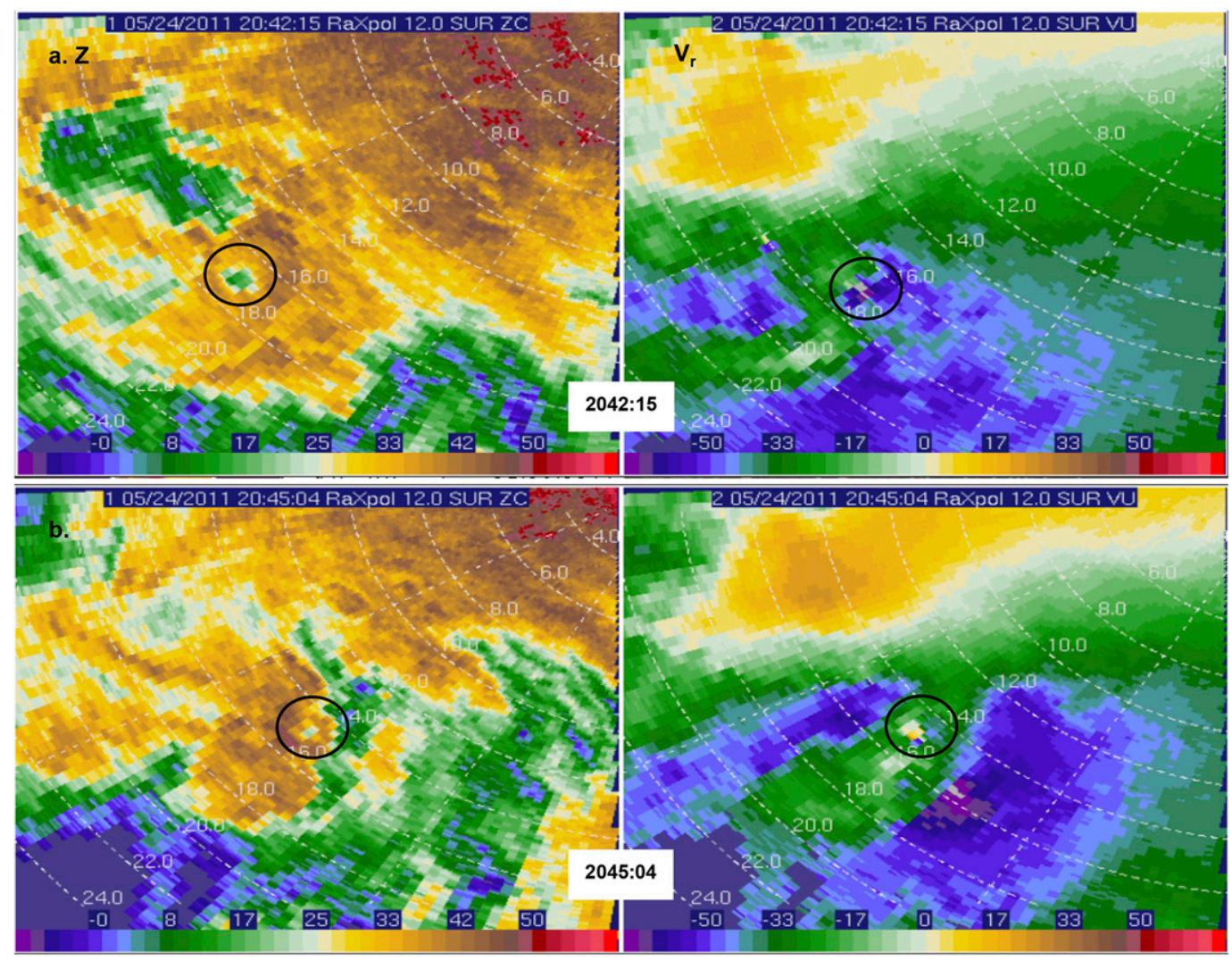

FIG. 3. (left) Reflectivity factor $(\mathrm{dB} Z)$ and (right) radial velocity $\left(\mathrm{m} \mathrm{s}^{-1}\right)$ for the $12^{\circ}$ elevation $(z \sim 3 \mathrm{~km}$ at $15-\mathrm{km}$ range) at (a) 2042:15 and (b) 2045:04 UTC. The tornadic circulation (circled in black) becomes removed from the maximum cyclonic shear associated with the new mesocyclone between these two times.

formation of tornado 2. Between 2044 and 2048 UTC, a narrow, crescent-shaped bounded weak echo region (BWER) developed (Fig. 2) aloft, in conjunction with a broadening area of high $Z_{\mathrm{DR}}$ above the freezing level, associated with a developing $Z_{\mathrm{DR}}$ column (Kumjian and Ryzhkov 2008). The development of these features suggests that the updraft intensified over this period. The BWER first became evident in the $18^{\circ}$ elevationangle observations $(z \sim 4.3 \mathrm{~km})$ around 2045:30 UTC. The timing of this feature is simultaneous with the RFGF occluding tornado 1 just prior to its decay (which began around 2045:30 UTC as well). By examining the vertical and horizontal spatial context of these features, it appears that the leading edge of the low-level RFGF evident in the low-level radial velocity field (not shown) (also collocated with the reflectivity gradient of the hook echo) is well matched with the rear edge of the BWER, which is located immediately north of the leading lowlevel RFGF (Fig. 8). The spatial correlation between these features is consistent with the notion of air being forced to ascend along the RFGF, acquiring a helical trajectory as it rises through the updraft. It is possible that the surging RFGF increased low-level convergence along its leading edge and triggered the updraft pulse.

\section{(ii) Low levels}

Between 2044 and 2045 UTC, a narrow, shallow (depth $\sim 2 \mathrm{~km}$ ) appendage of outbound radial velocities northwest of the tornadic circulation at low levels $(z \sim 1 \mathrm{~km})$ began to progress into the hook region (Fig. 4, black arrow). This extrusion of radar-relative outbound velocities extended rearward from the forward-flank region and was associated with strengthening cyclonic shear about $4 \mathrm{~km}$ to the northwest of the decaying tornado 1 , which was no longer associated with a low-level mesocyclone. The development of a new low-level mesocyclone (which would later be associated with the genesis of tornado 2) subsequently occurred in this region to the northwest of the remnants of tornado 1 (Fig. 9). This velocity feature also marked the leading edge of another surge of high radar reflectivity moving from the rear of the forward flank through the hook echo. Because vertical motions are not retrievable, it is unclear whether this feature was associated with a rear-flank downdraft. However, as it passed 


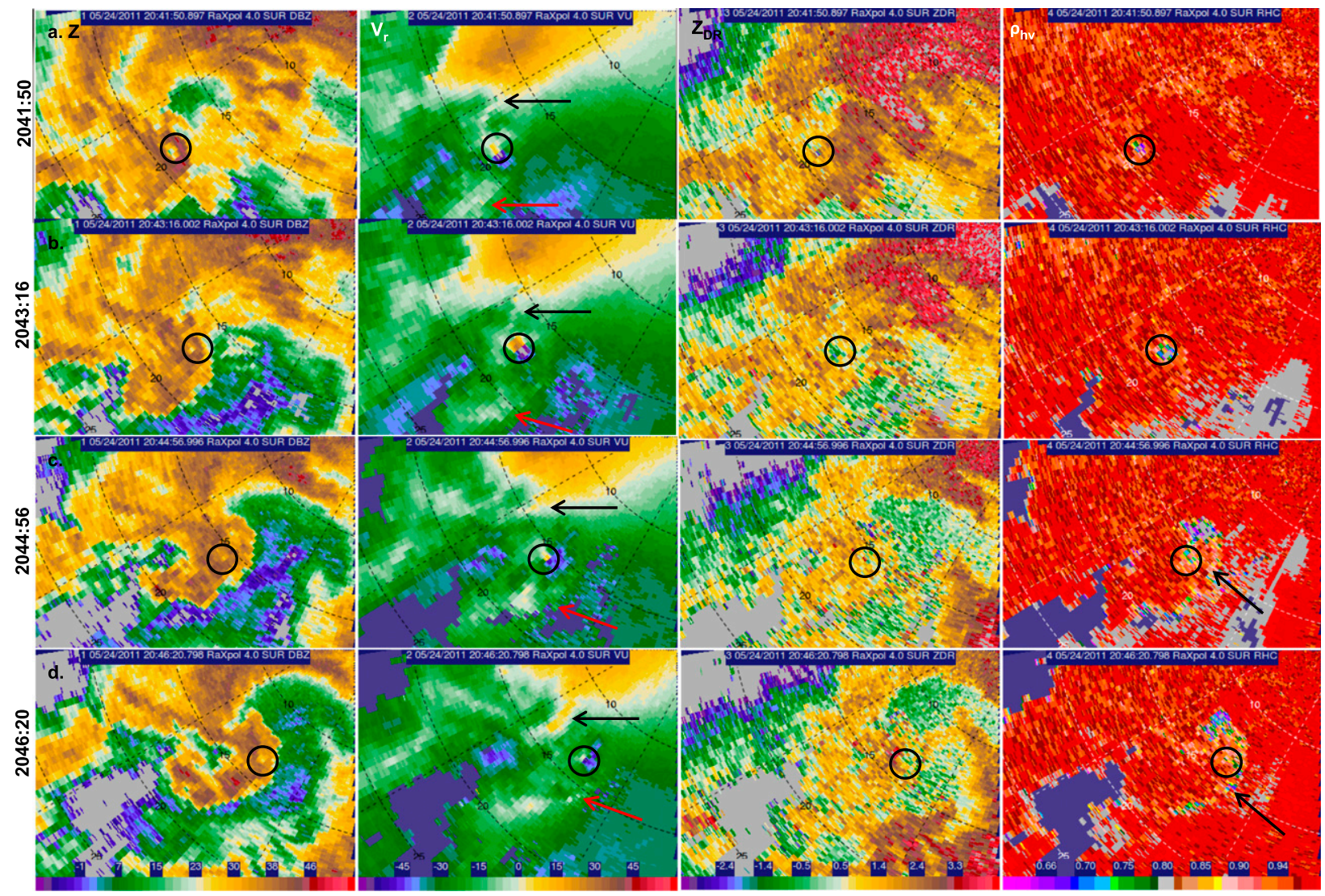

FIG. 4. Evolution of tornado 1 and RFGFs at $4^{\circ}$ elevation angle $(z \sim 1 \mathrm{~km})$. Columns are as in Fig. 2, but for (a) 2041:50, (b) 2043:16, (c) 2044:56, and (d) 2046:20 UTC. Black circles indicate the locations of tornado vortex, red arrows indicate the location of wind shift associated with the primary RFGF, the black arrows indicate the outbound velocity appendage associated with a secondary RFGF surge in the $V_{r}$ panels, and denote the location of reduced $\rho_{\mathrm{hv}}$ along the RFGF. Range rings are every $5 \mathrm{~km}$.

through the hook echo, it behaved like a secondary rearflank momentum surge (Lee at al. 2011, 2012; Kosiba et al. 2013; Skinner et al. 2014) and will be referred to as such hereafter.

At 2048 UTC, there were two RFGFs apparent in the low-level radial velocity imagery (Figs. 9 and 10). The primary RFGF was located along the leading edge of the high reflectivity gradient bounding the hook echo; the secondary RFGF was to the northwest. Between 2047 and 2049 UTC, the remnants of the earlier tornadic circulation, before becoming indiscernible amidst the background vorticity, were wrapped back along the original RFGF toward the new developing low-level mesocyclone (Fig. 10, red circle) that was associated with the secondary RFGF. The secondary RFGF continued surging southeastward, merging with the original RFGF. As this happened, the low-level vortex couplet associated with the nascent new low-level mesocyclone that would soon produce tornado 2 contracted in size and strengthened (Fig. 11, left column).
The organization and intensification of the rotation that ultimately produced tornado 2 occurred first at the lowest elevation angle $\left(4^{\circ}, z \sim 800 \mathrm{~m}\right.$ AGL), with the development of the previously mentioned low-level mesocyclone (Fig. 11, left column). Prior to tornadogenesis, there was a noticeable difference between how rotation evolved below $1.5 \mathrm{~km}$ and above this height. In the lowest two elevation angles, the low-level mesocyclone contracted, intensified, and organized over a $\sim 3$-min period from 2048 to 2051 UTC. A similar steady intensification was not apparent at higher elevation angles. Despite the strengthening low-level rotation, the mesocyclone aloft remained broad and diffuse. At 2051: 01 UTC, strong rotation suddenly appeared in the upper elevation scans $(2<z<3.25 \mathrm{~km})$ (Fig. 11c, right column), without prior indication of significant intensification. Within $\sim 30$ s (i.e., two volume scans) of this observation, the rotation had intensified such that the tornadic-velocity threshold was crossed at all elevation angles except $10^{\circ}(z \sim 1.5 \mathrm{~km})$, and tornado damage was 
a.

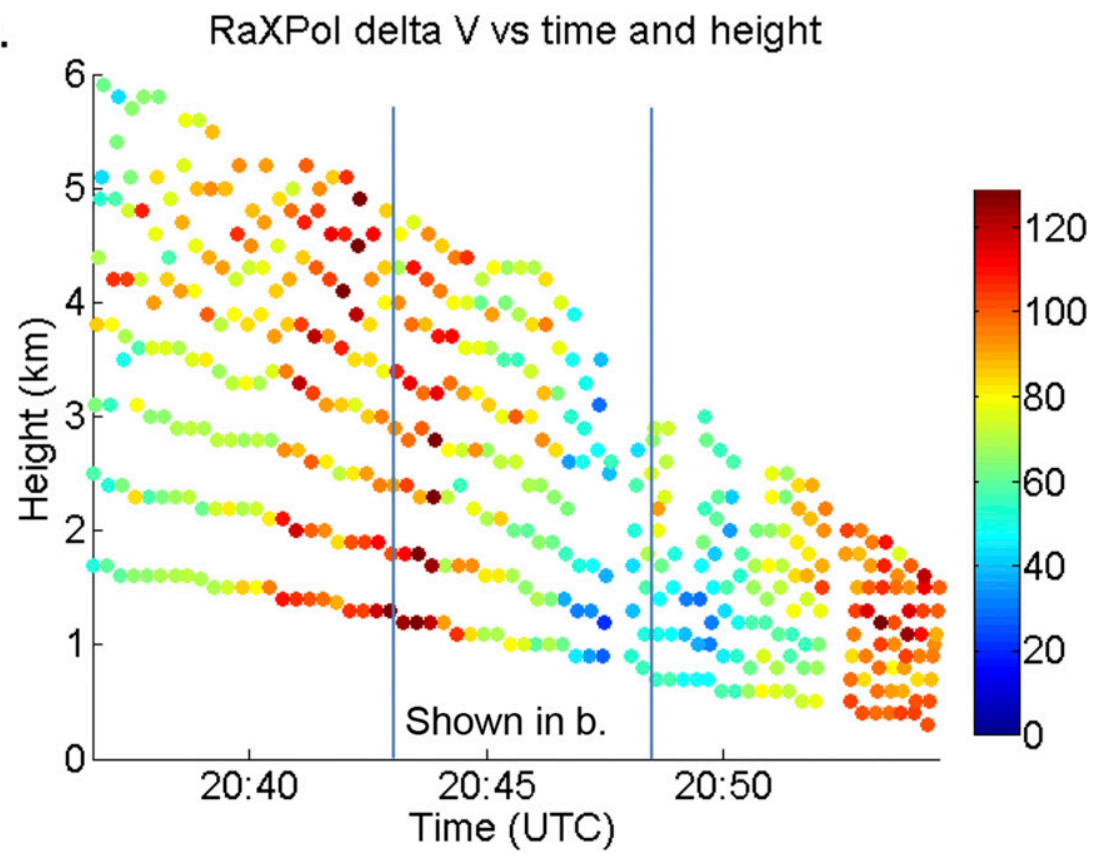

b.

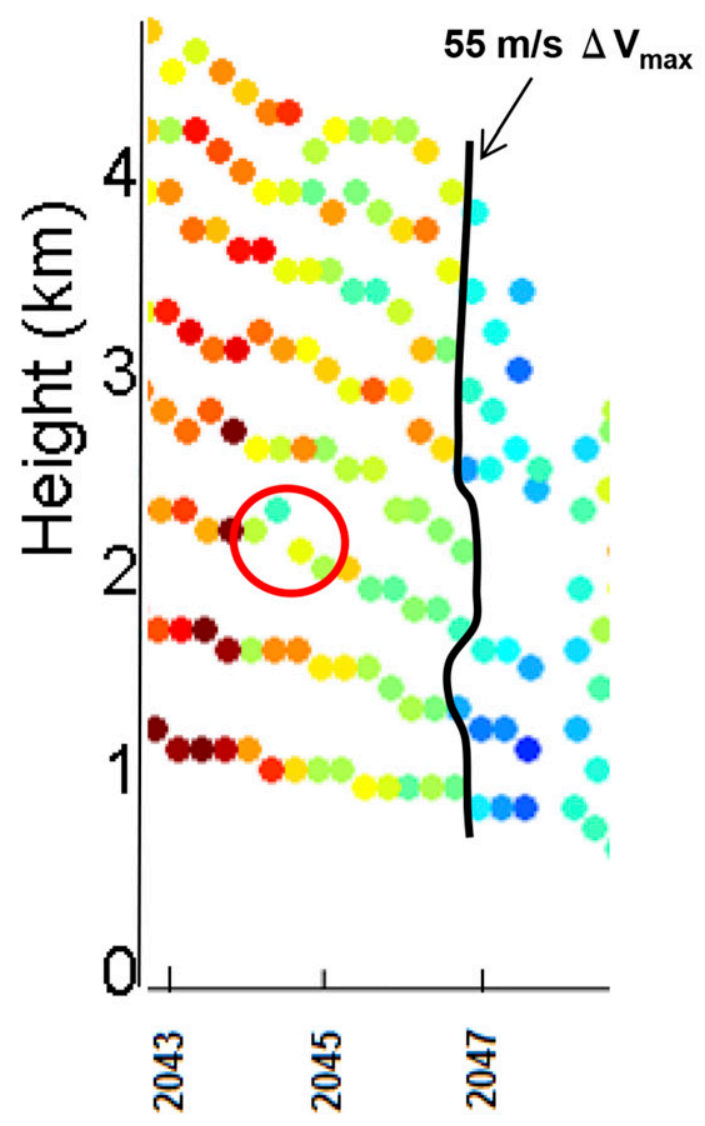

FIG. 5. Plot of $\Delta V_{\max }$ with time (UTC, abscissa, increasing to the right), and height (km, ordinate, increasing up) (a) for the entire duration of the volumetric data collection between 2036 and 2055 UTC. The magnitude of $\Delta V_{\max }$ is indicated by the color $\left(\mathrm{m} \mathrm{s}^{-1}\right)$. Vertical lines indicate portion of graphic zoomed in for (b). (b) As in (a), but zoomed in on tornado decay ( 2043-2048 UTC). The ellipse indicates the region of initial weakening at midlevels. 


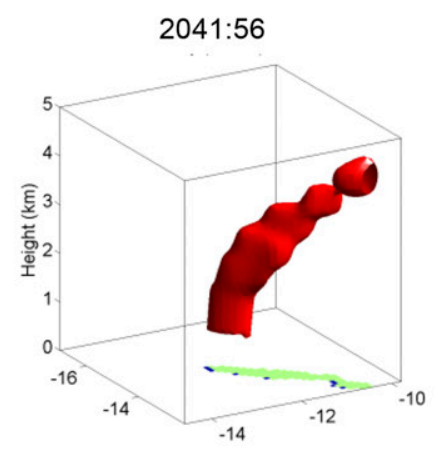

2044:12

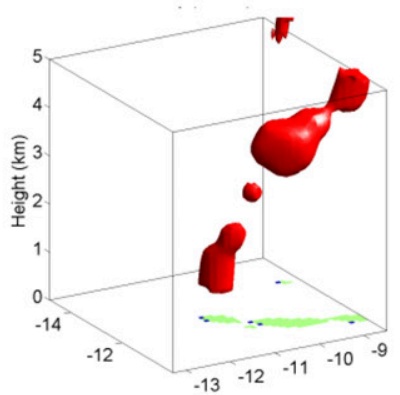

2046:09

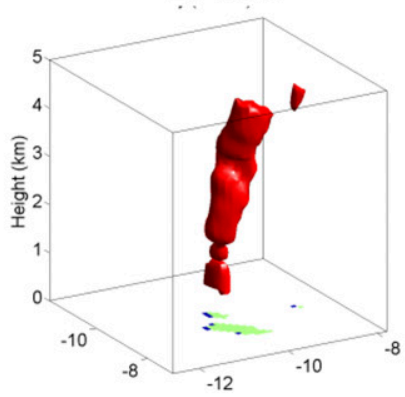

2047:00

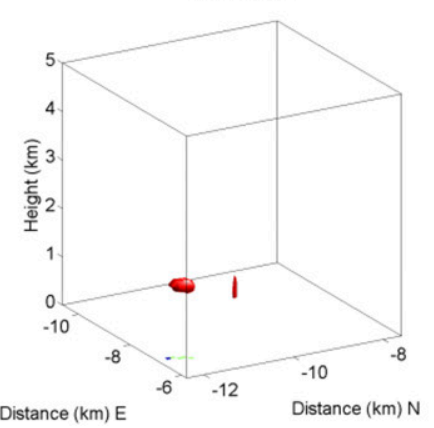

$2042: 47$



2045:02

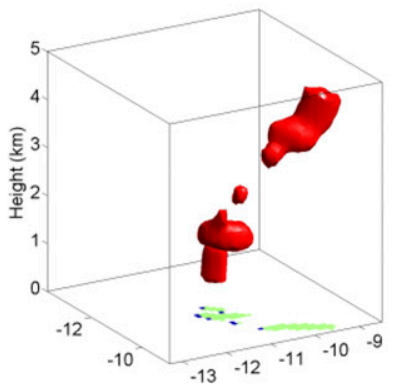

2046:26
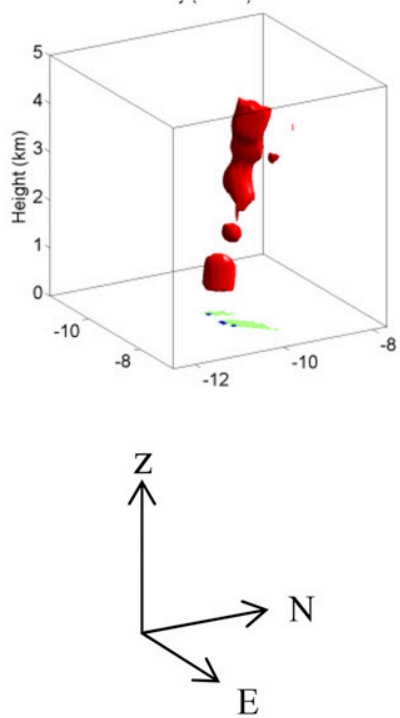

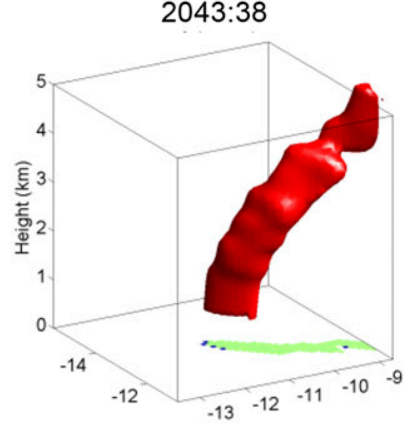

2045:53

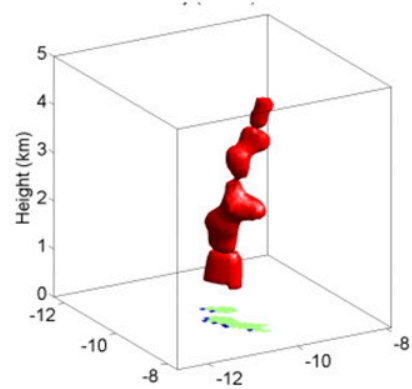

2046:43



FIG. 6. Pseudovorticity isosurfaces bound by the $0.15 \mathrm{~s}^{-1}$ contour and shadow projection (green shading) on the $x-y$ plane over the duration of tornado 1 for which data are available. Times indicated are in UTC.

observed. The weakness in rotation at $10^{\circ}$ will be addressed in section 4.

From the radar data alone, it is unclear what prompted the sudden intensification of rotation aloft immediately prior to tornadogenesis. It is also unclear what the exact cause and effect relationship was between the preexisting low-level rotation and the sudden appearance of strong rotation around $\sim 2.5 \mathrm{~km}$. 


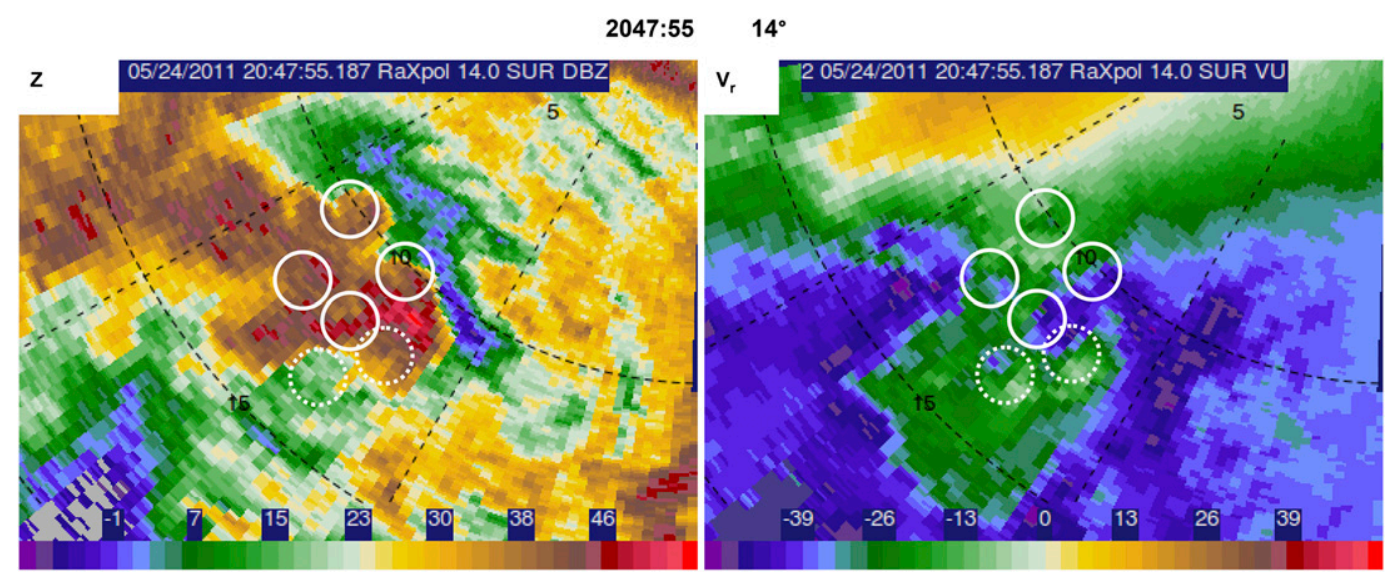

FIG. 7. (left) Reflectivity $(\mathrm{dBZ})$ and (right) radial velocity $\left(\mathrm{m} \mathrm{s}^{-1}\right)$ of midlevel $(z \sim 3 \mathrm{~km})$ rotation at 2047:55 UTC (after the dissipation of tornado 1 and before genesis of tornado 2). Prominent misocyclone (solid) and miso-anticyclone (dotted) vortex signatures in the hook echo are circled.

The observations seem to suggest, however, that the appearance of strong rotation aloft represents a critical point in the tornadogenesis process for this particular case. The observed sequence of events may imply that there needs to be a collocation of strong rotation at low levels with strong rotation at higher levels $(z>2 \mathrm{~km})$ to form a tornado, since there was strong low-level rotation prior to tornadogenesis and yet no tornado formed. Perhaps the onset of strong rotation aloft was required in this case to overcome the stability associated with an environmental inversion (see section 4) by generating a dynamically induced upward-directed pressure gradient force, allowing parcels to be stretched through the inversion rather than being capped by it.

\section{2) TORNADO-SCALE EVOLUTION}

During the $2 \mathrm{~min}$ centered on tornadogenesis (i.e., 2051 UTC), $\Delta V_{\max }$ increased from $\leq 45$ to $>70 \mathrm{~m} \mathrm{~s}^{-1}$ at nearly all heights (Fig. 12). Thus, rotation increased nearly simultaneously over the depth data were collected, with velocities achieving tornadic strength at all heights over the observable domain within $30 \mathrm{~s}$ of each other. There was no evidence that the onset of tornadic rotation built downward from aloft; there was no descending TS. ${ }^{9}$ Rather, tornadogenesis occurred as $\Delta V_{\max }$ intensified nearly simultaneously over the depth of the

\footnotetext{
${ }^{9}$ According to Trapp et al. (1999), a descending vortex signature associated with the DPE will take upward of 10 min to descend to the surface from midlevels. Thus, it is clear that the temporal resolution of this dataset is more than sufficient to unambiguously resolve a descending TVS (TS).
}

sample domain above the location of preexisting low-level rotation.

Once genesis occurred, the condensation funnel quickly grew in width. During the 4-min period, between 2051:00 ( $~ 10$ s prior to tornadogenesis) and 2055:10 UTC, what started out as a low-hanging wall cloud transitioned into a large "wedge" tornado (Fig. 13). Despite the rapid visual growth of the tornado, the initial intensification process was not uniform with height. There was a relative minimum in $\Delta V_{\max }$ around $\sim 1.5 \mathrm{~km}$ (Fig. 12), slightly below the layer in which the initial decay of the first tornado was noted earlier. Above this height, the rotation intensified more rapidly than below it. When data collection resumed after a brief break at 2052 UTC to change the scanning strategy, all $\Delta V_{\max }$ values were similarly intense and continued to increase through the end of the volumetric data coverage at 2055 UTC.

To view the evolution of tornado 2's rotation in three dimensions, the $0.2 \mathrm{~s}^{-1}$ pseudovorticity contour was used instead of the $0.15 \mathrm{~s}^{-1}$ contour because the $0.15 \mathrm{~s}^{-1}$ threshold was not consistent with the times when a tornado was known to have been occurring (Fig. 14). Because the storm was closer to the radar at this time, the spatial resolution was higher and smaller-scale wind maxima were better sampled, generally allowing for higher vertical vorticity values to be calculated. Just prior to the genesis of tornado 2 , the low-level vorticity began to increase and reach tornadic intensity (2050:21 UTC) (Fig. 14). It briefly weakened below the $0.2 \mathrm{~s}^{-1}$ threshold at 2050:54 UTC; however, at this time vorticity aloft suddenly increased to tornadic intensity. Within one 17-s volume scan (between 2050:54 and 2051:11 UTC), vorticity $>0.2 \mathrm{~s}^{-1}$ developed throughout almost the entire depth of data collection (through $\sim 3 \mathrm{~km}$ ARL). The 




FIG. 8. Relationship between RFGF and BWER for (left) the $4^{\circ}$ elevation angle at 2047:45 UTC and (right) the $18^{\circ}$ elevation angle at 2047:41 UTC. (a) Reflectivity factor (dBZ), (b) $Z_{\mathrm{DR}}$ (dB), and (c) $\rho_{\mathrm{hv}}$, with overlays of the BWER (black ellipsoid), leading edge of the hook echo (black curve), and an indication of the location of the melting layer (yellow curve). 


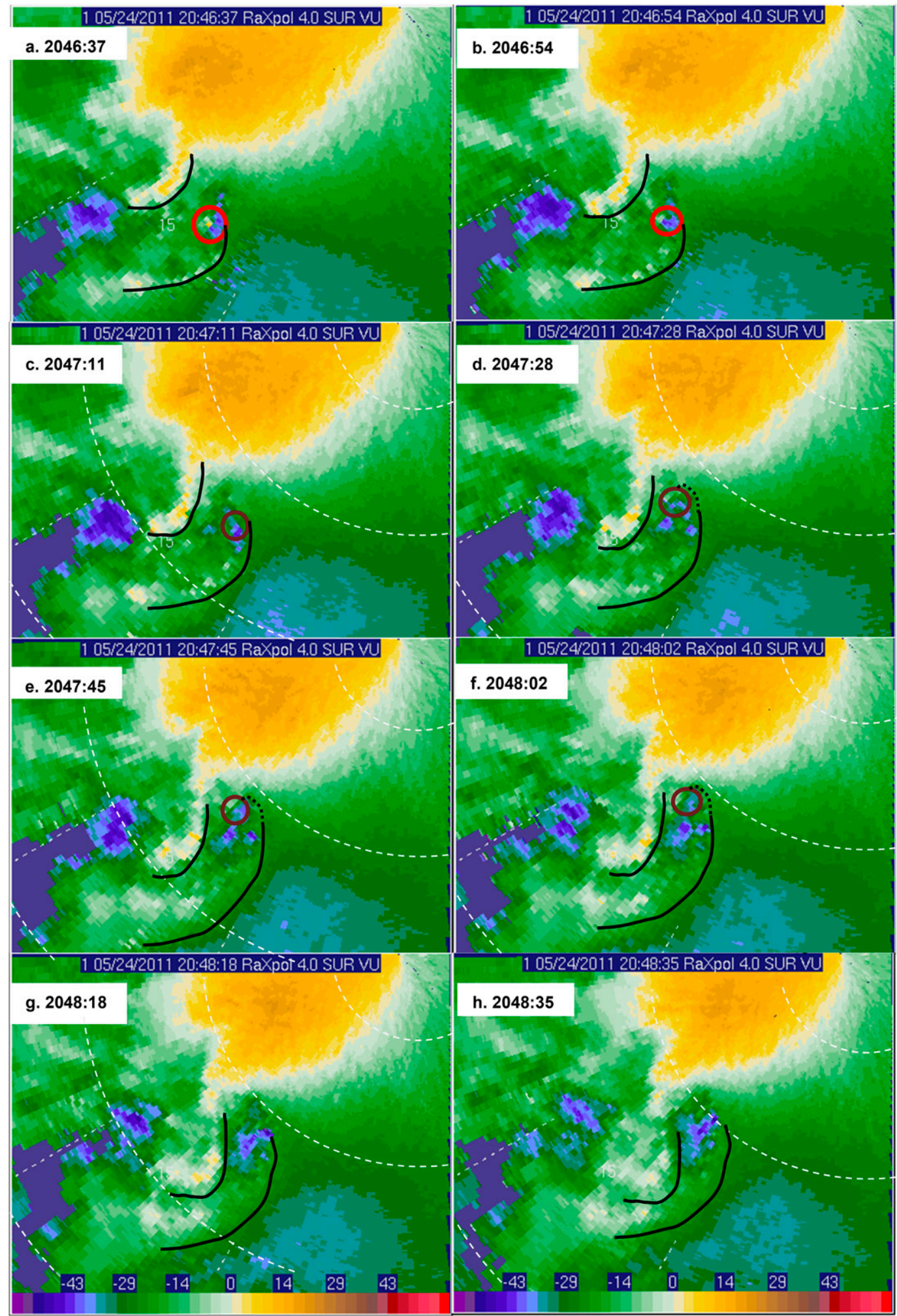

FIG. 9. (a)-(h) Evolution of low-level rotation from radial velocity $\left(\mathrm{m} \mathrm{s}^{-1}\right)$ at the $4^{\circ}$ elevation angle prior to tornadogenesis. Images are every $17 \mathrm{~s}$ from 2046:37 to 2048:35 UTC. Red (brown) circles indicate the tornadic (remnant) circulation from tornado 1. Only the rotation associated with tornado 1 is denoted. Black lines in (a) denote RFGF locations. Range rings are 5, 10, 15, and $20 \mathrm{~km}$. 


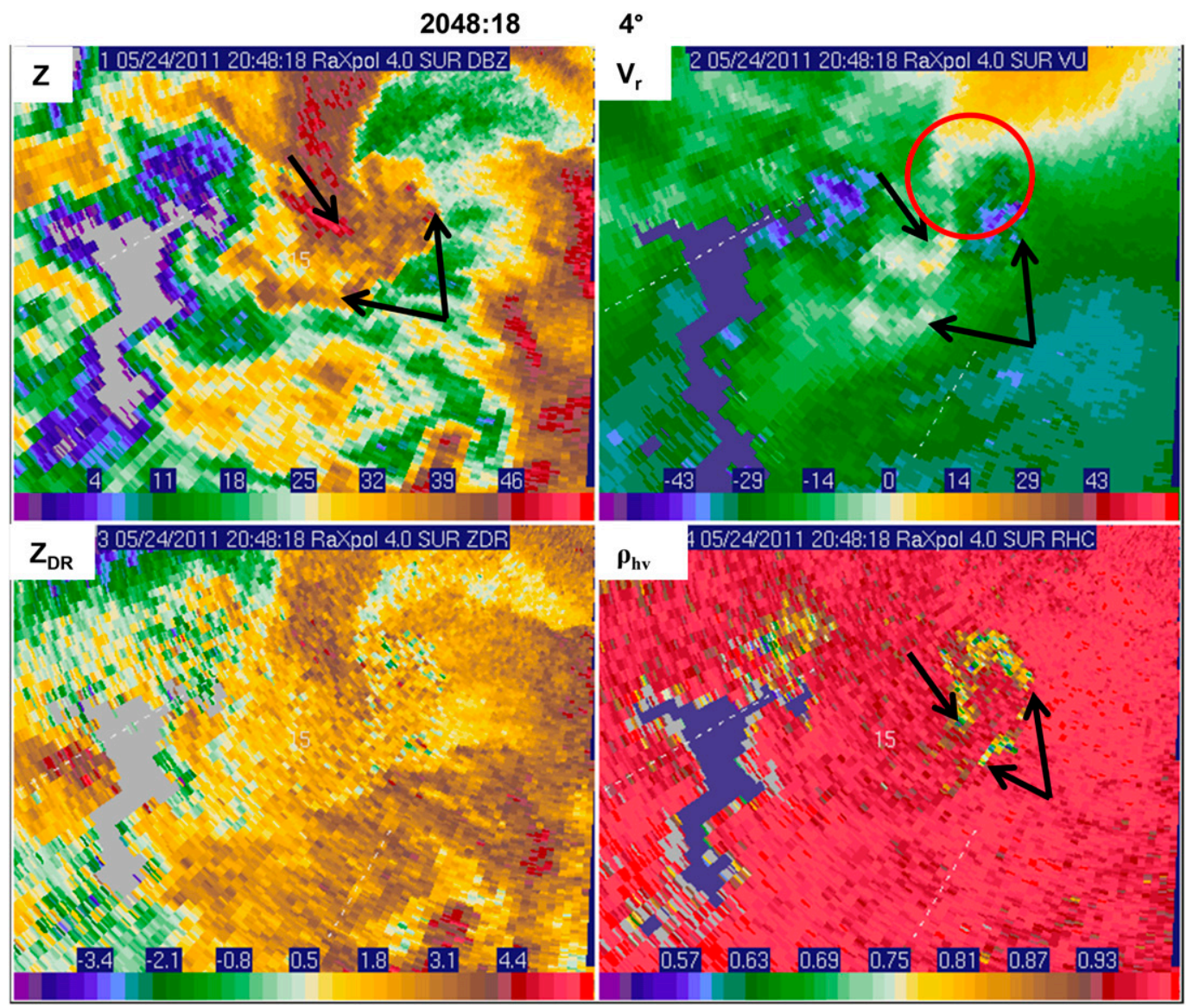

FIG. 10. The four-panel image of the secondary RFGF surge at 2048:18 UTC from the $4^{\circ}$ elevation angle. RFGFs are indicated by black arrows, and the developing low-level mesocyclone is denoted by the red circle.

exception to this was the shallow layer mentioned in the previous section between $\sim 1.5$ and $2 \mathrm{~km}$ that did not intensify simultaneously. A relative minimum in the strength of rotation in this layer is evident through 2052:01 UTC. These observations again are consistent with the behavior of $\Delta V_{\max }$.

\section{Discussion}

\section{a. Tornado decay}

Tornado 1's TS at all elevation angles reached the subtornadic criterion $\left(\Delta V_{\max }<55 \mathrm{~m} \mathrm{~s}^{-1}\right)$ between the same two volume scans, occurring concurrently over the depth of the data domain within 30s (Figs. 5 and 6). However, the complete dissipation of the subtornadic velocity couplet did not behave in this way. After $\Delta V_{\max }$ dropped below $55 \mathrm{~m} \mathrm{~s}^{-1}$, the subtornadic velocity couplet first disappeared at $2.5 \mathrm{~km}$, then above this height. The subtornadic low-level couplet persisted for several volume scans after tornado cessation, finally completely dissipating about a minute later. The observation of $v e$ locity couplet behavior with height is consistent with what F13 found in their rapid-scan study of four tornadic TVSs (resolution was lower so TSs could not be resolved).

From the observations presented above, there is a variation in behavior of tornado "dissipation" depending upon the threshold of shear across the velocity couplet used to define the TS. For the purposes of this study, the velocity couplet was considered tornadic when $\Delta V_{\max }>$ $55 \mathrm{~m} \mathrm{~s}^{-1}$. The reader is reminded that this value was selected based upon the times when the NWS damage survey (NWS 2011c) specified there was a tornado. The velocity couplet at all heights persisted longer than the reported tornado (Fig. 5). If the tornado were defined by a different value of $\Delta V_{\max }$, then the decay of the TS would not have matched the inferred behavior of the actual tornado demise. To illustrate this point, consider what was observed in this case, using a criterion of $\Delta V_{\max } \geq$ $55 \mathrm{~m} \mathrm{~s}^{-1}$. By this definition, the tornado decayed nearly simultaneously, within $\sim 30$ s at all elevations. However, if the damage survey had not been available, and a 


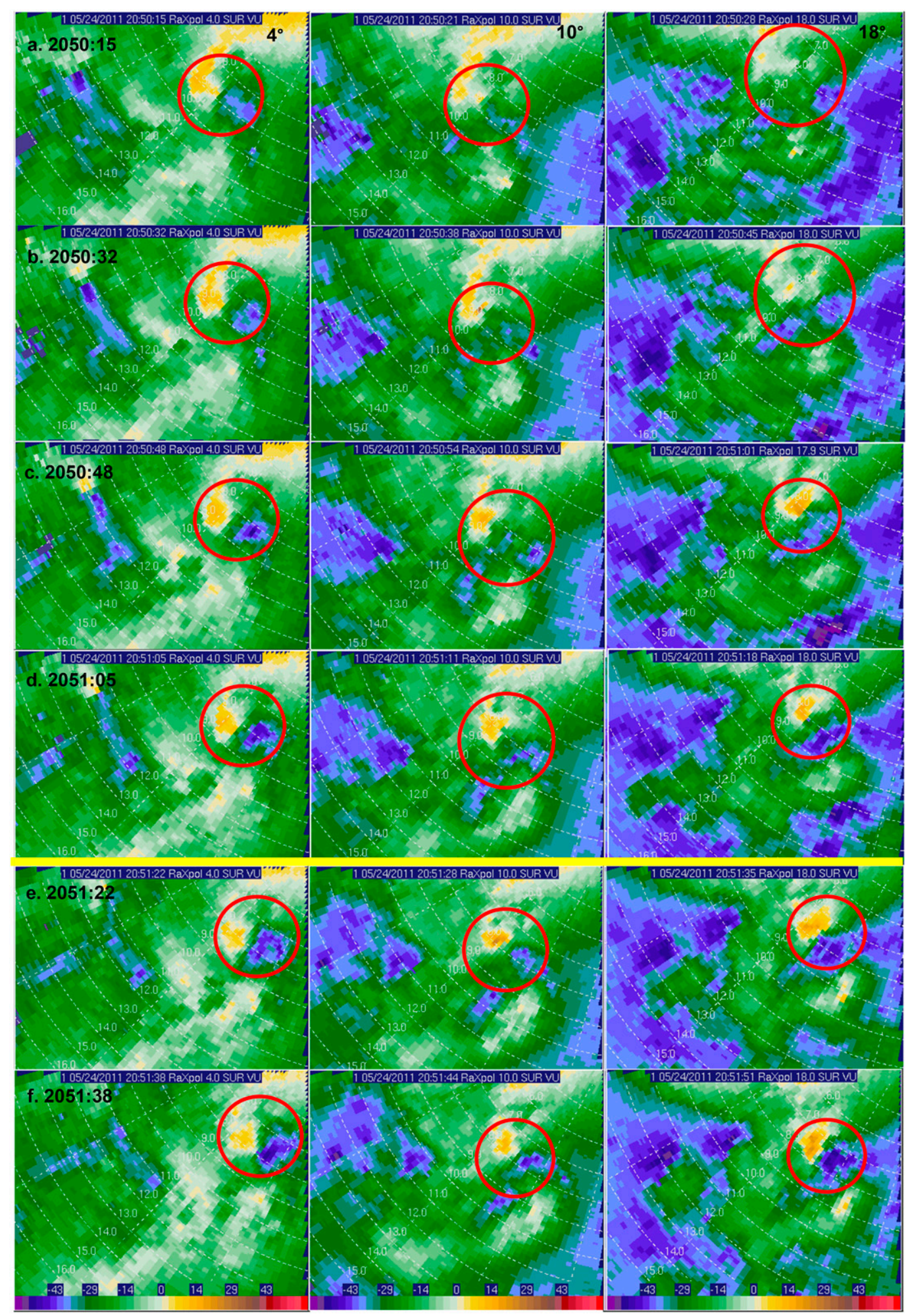

FIG. 11. Evolution of Doppler velocity $\left(\mathrm{m} \mathrm{s}^{-1}\right)$ at increasing elevation angles every $17 \mathrm{~s}$ from (a) 2050:15 to (f) 2051:35 UTC. (left) The $4^{\circ}$ elevation angle, (middle) $10^{\circ}$ elevation angle, and (right) $18^{\circ}$ elevation angle. Tornadogenesis occurred around 2051:20 UTC. The thick yellow line indicates the time of tornadogenesis. Red circles denote general regions of rotation associated with the tornado or pretornadic vortex. Range rings are shown every $1 \mathrm{~km}$; the tornado developed $\sim 9 \mathrm{~km}$ to the southwest of RaXPol. 
a.

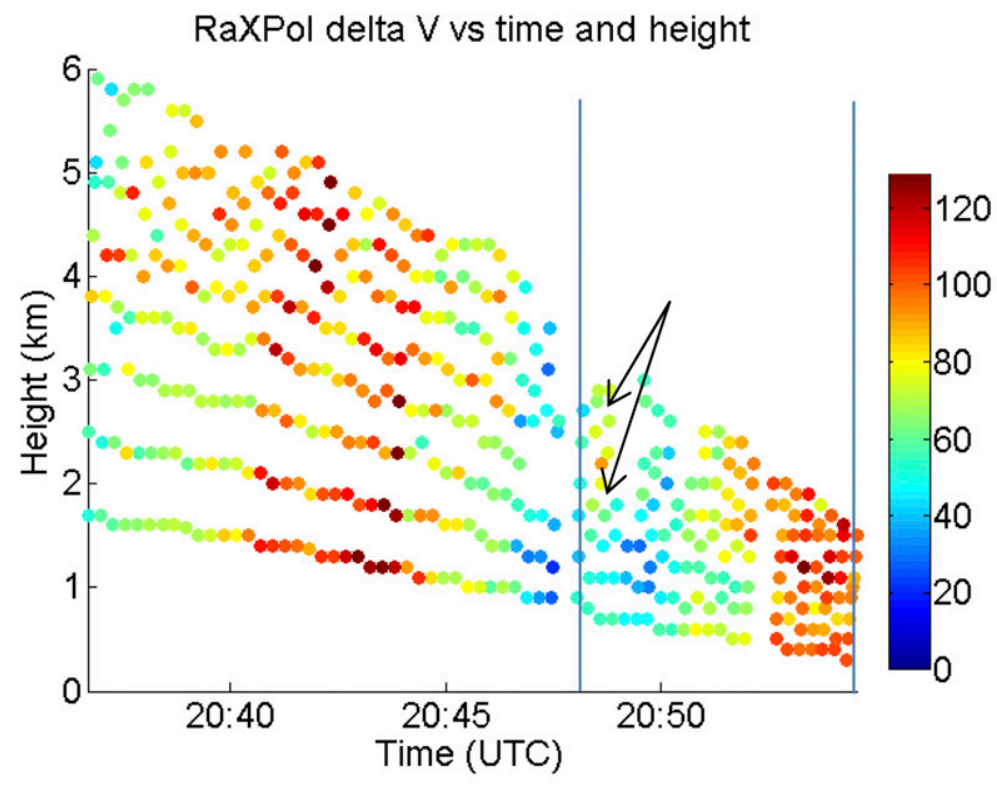

b.

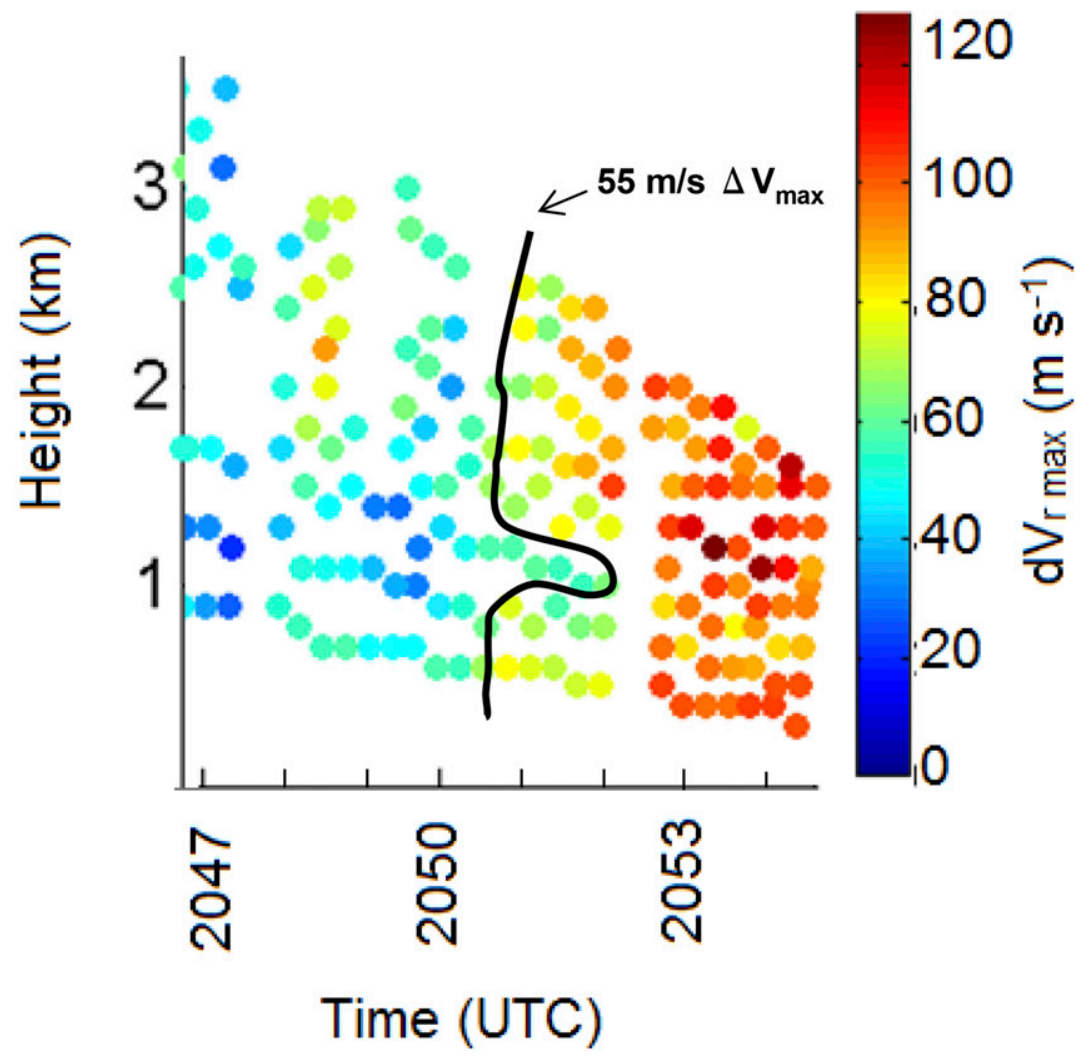

FIG. 12. (a),(b) As in Fig. 5, but (b) is zoomed in on tornadogenesis (2047-2055 UTC) [blue lines in (a)]. In (a), arrows point to increased circulation aloft that did not become tornadic. Tornadogenesis occurred between the 2051:11 and 2051:28 UTC scans.

threshold of $40 \mathrm{~m} \mathrm{~s}^{-1}$ was used instead, the tornado would have appeared to decay first at midlevels, then aloft, and last at the surface. This observation implies that the dissipation tendency of a "TS" is contingent upon the threshold one uses to define a TS. If one applies a TS threshold too liberally, a TS may exist without a tornado; however if a TS threshold is applied too conservatively, the actual evolution of the rotation may be overlooked. 


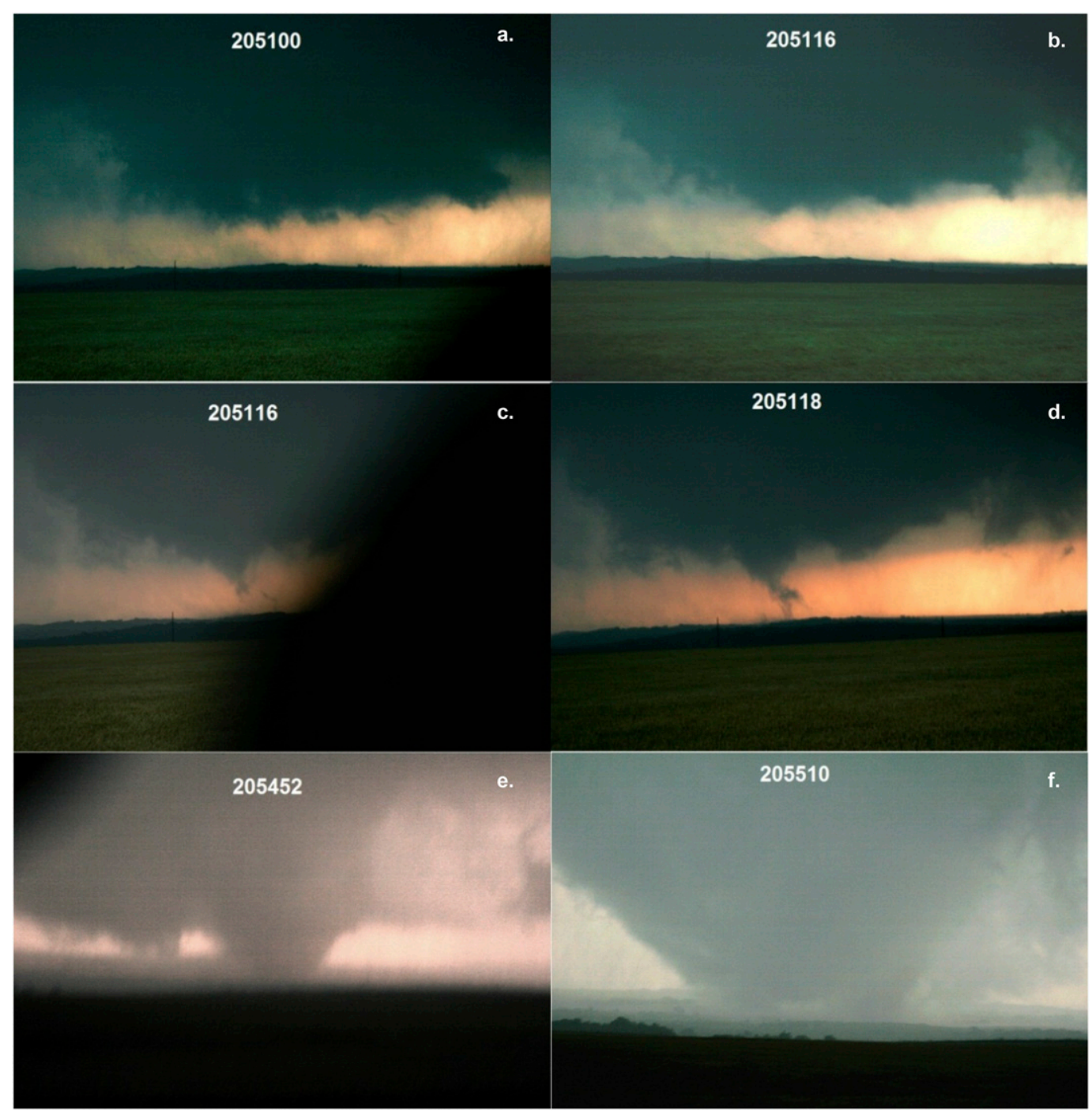

FIG. 13. Visual observations of the developing tornado taken from the RaXPol deployment location. (Courtesy of J. Houser.)

\section{b. Storm-scale processes during decay and storm cycling}

Because this storm produced two tornadoes and had two midlevel mesocyclones for a time, its evolution can be compared to conceptual models of cyclic mesocyclogenesis/tornadogenesis (Burgess et al. 1982; Adlerman et al. 1999; DB02a; Beck et al. 2006). The traditional paradigm of tornado decay for cyclic and noncyclic tornadoes involves a surge of outflow, typically associated with the rear-flank downdraft, resulting in an occlusion of outflow around the tornado (Brandes 1978; Lemon and Doswell 1979; Brooks et al. 1993; Davies-Jones et al. 2001; Marquis et al. 2012). The surge of outflow is attributed to a weakening or reversal of the vertical pressure gradient force near the cloud base (Wicker and Wilhelmson 1995). The occlusion cuts the tornado off from its source of warm, buoyant inflow, causing the supporting updraft to weaken, which reduces convergence of high angular-momentum air into the vortex, causing it to decay. It has been observed that dissipating tornadoes move rearward relative to the updraft motion, sometimes nearly making a full loop (Burgess et al. 1982; DB02a; Bluestein et al. 2010; Tanamachi et al. 2012), particularly when supercells undergo cyclic mesocyclogenesis. DB02a found that this behavior occurs when tornadoes become decoupled from the parent updraft and are advected rearward with the outflow air circulating around the mesocyclone. A factor in this process is weakening inflow. Prior to the 


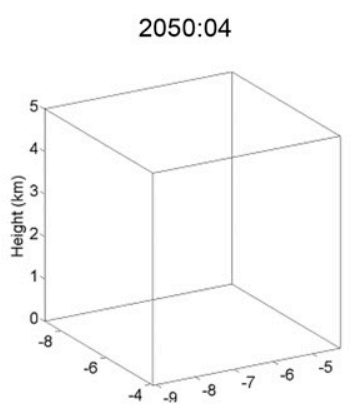

2050:54

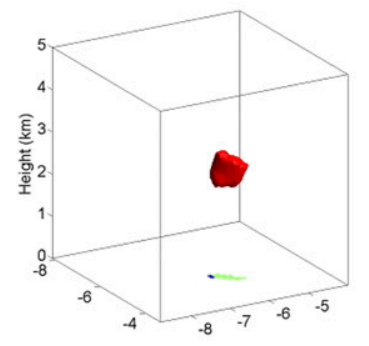

2051:44

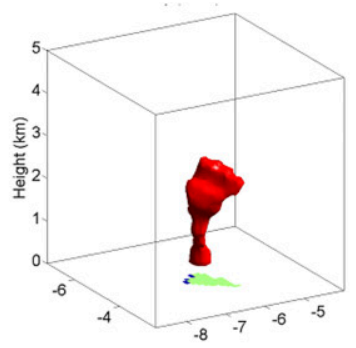

2053:01



2053:50

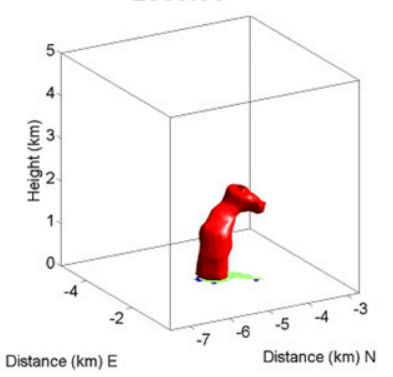

2050:21

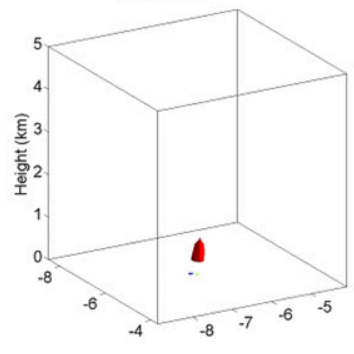

2051:11

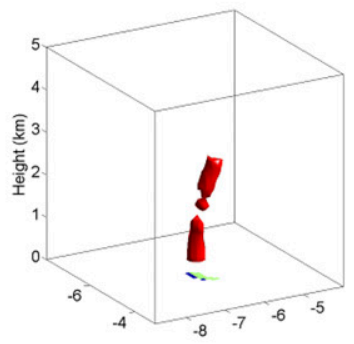

2052:01



2053:17

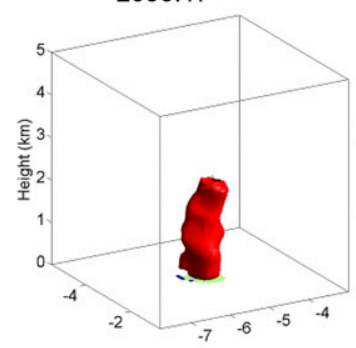

2054:07

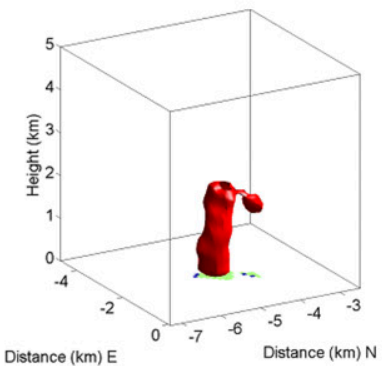

2050:38

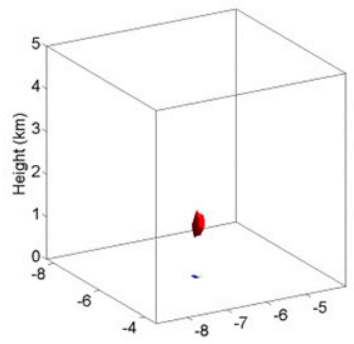

2051:28

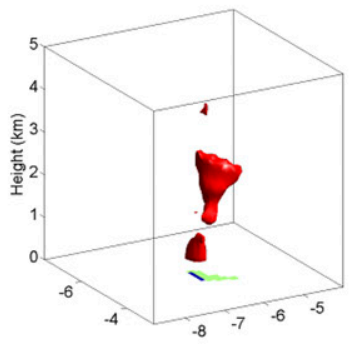

2052:45
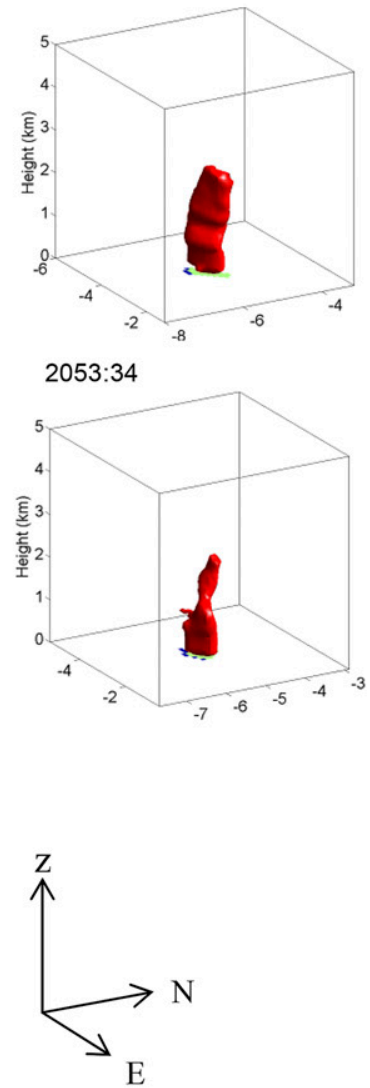

FIG. 14. As in Fig. 6, but for the $0.2 \mathrm{~s}^{-1}$ isosurface during genesis of tornado 2 (2050:54-2054:07 UTC). 
occlusion, strong low-level inflow is able to confine the RFGF and inhibit it from wrapping around the mesocyclone; but as inflow weakens, it is no longer able to contain the RFGF.

In this case, it appears that tornado 1 was occluded by outflow, but the subsequent evolution during its demise was atypical. As in the traditional decay paradigm, it appears that storm-relative inflow weakened (reasons for this are outside the scope of this paper), which allowed the RFGF to surge eastward, advecting the tornado away from the updraft. A separation between the low-level tornado and the updraft resulted and was an effect of differential horizontal motion between the two. These observations support two primary conclusions drawn by Dowell and Bluestein (2002b): in order for a tornado to be maintained, (i) some degree of balance must exist between the inflow and outflow; and (ii) the tornado's motion vector must be nearly parallel to that of the updraft. However, unlike the traditional decay or cyclic supercell models, the tornado was not advected rearward around the mesocyclone to the left of the updraft motion vector, but rather downstream and to the right from the low and midlevel mesocyclone and updraft, toward the leading edge of the RFGF. This behavior of the low-level vortex during tornado decay is similar to the nonoccluding cyclic mesocyclone (NOCM) mode discussed by Adlerman and Droegemeier (2005).

Just prior to the time when the low-level circulation was advected toward the leading edge of the RFGF, a new midlevel mesocyclone formed to the southeast of the original mesocyclone. The new mesocyclone is presumed to be associated with an updraft pulse, which was first inferred by the appearance of a new partial $Z_{\mathrm{DR}}$ ring and, several minutes later, by the formation of a $Z_{\mathrm{DR}}$ column and a BWER. Concurrently, the RFGF began surging outward to the east and southeast, away from the updraft. The midlevel observations thus far are in good agreement with the Kumjian et al. (2010) study of polarimetric features of cyclic supercells and are similar to the classic cyclic supercell models and explanations given by Burgess et al. (1982), DB02a, Beck et al. (2006), French et al. (2008), and the occluding cyclic mesocyclone (OCM) mode of Adlerman and Droegemeier $(2002,2005)$.

In this case, however, the original mesocyclone remained the dominant mesocyclone for nearly $6 \mathrm{~min}$ after the new mesocyclone formed. After the original mesocyclone weakened, midlevel rotation became very broad and the new mesocyclone did not further intensify. Instead, it dissolved into the larger-scale rotation during a period of reorganization. Another difference is that the tornado transitioned from being associated with the original mesocyclone, to being rooted in the new mesocyclone as it was advected away from the original updraft by the surge in rear-flank outflow. The RFGF did not surge into the inflow, allowing the "new," and later the reorganizing, mesocyclone to have continued access to presumably buoyant or less negatively buoyant inflow air. ${ }^{10}$ Thus, it appears that the storm-scale evolution occurring during the interim between tornado 1 and tornado 2 is a type of failed cyclic mesocyclone/decay process that, to the authors' knowledge, has not been documented before. The tornado behaved in a manner consistent with the NOCM, while the mesocyclone attempted to cycle similarly to the OCM mode of Adlerman and Droegemeier (2005).

Storm-scale processes also appeared to impact the genesis of tornado 2. A secondary RFGF surge occurred prior to tornado 2's genesis. A new low-level mesocyclone formed as this RFGF intersected the original RFGF, and about 2 min after this, tornadogenesis occurred. It is possible that when the secondary RFGF overtook the original RFGF, low-level convergence was augmented, which would be associated with an increase in upward acceleration that would act to stretch the broad field of preexisting low-level rotation forming an organized low-level mesocyclone. Kosiba et al. (2013) also observed a similar sequence of events for a different case and justified the enhancement of low-level vorticity using a dual-Doppler analysis.

It is important to note that vertical motions cannot be directly inferred from the single-Doppler observations. Speculation for locations of updrafts and downdrafts is based on the presence and locations of storm-scale features known to be associated with vertical motion (e.g., surges in rear-flank outflow, BWERs, $Z_{\mathrm{DR}}$ columns, etc.). Thus, the sequence of events described above is based upon the best information available and may not be entirely representative of the processes that occurred in reality. For this reason, a data assimilation modeling study would be very useful to diagnose the full threedimensional wind field and the dynamics of the storm.

A summary of the evolution of the cycling process described above with respect to the various storm-scale and tornado features is given in Fig. 15. Initially, $\left(t_{1}\right)$ there is a single midlevel mesocyclone. The low-level TS is located near the tip of the hook echo, just behind the primary RFGF. Storm-relative inflow is relatively strong. The second midlevel mesocyclone then separates from the original one and the low-level TS begins to be pushed down the primary RFGF in response to strengthening

\footnotetext{
${ }^{10}$ Storm-scale thermodynamic data are not available, but it is assumed that the environmental inflow into the storm was warmer and, therefore, more buoyant/less negatively buoyant than the outflow air.
} 

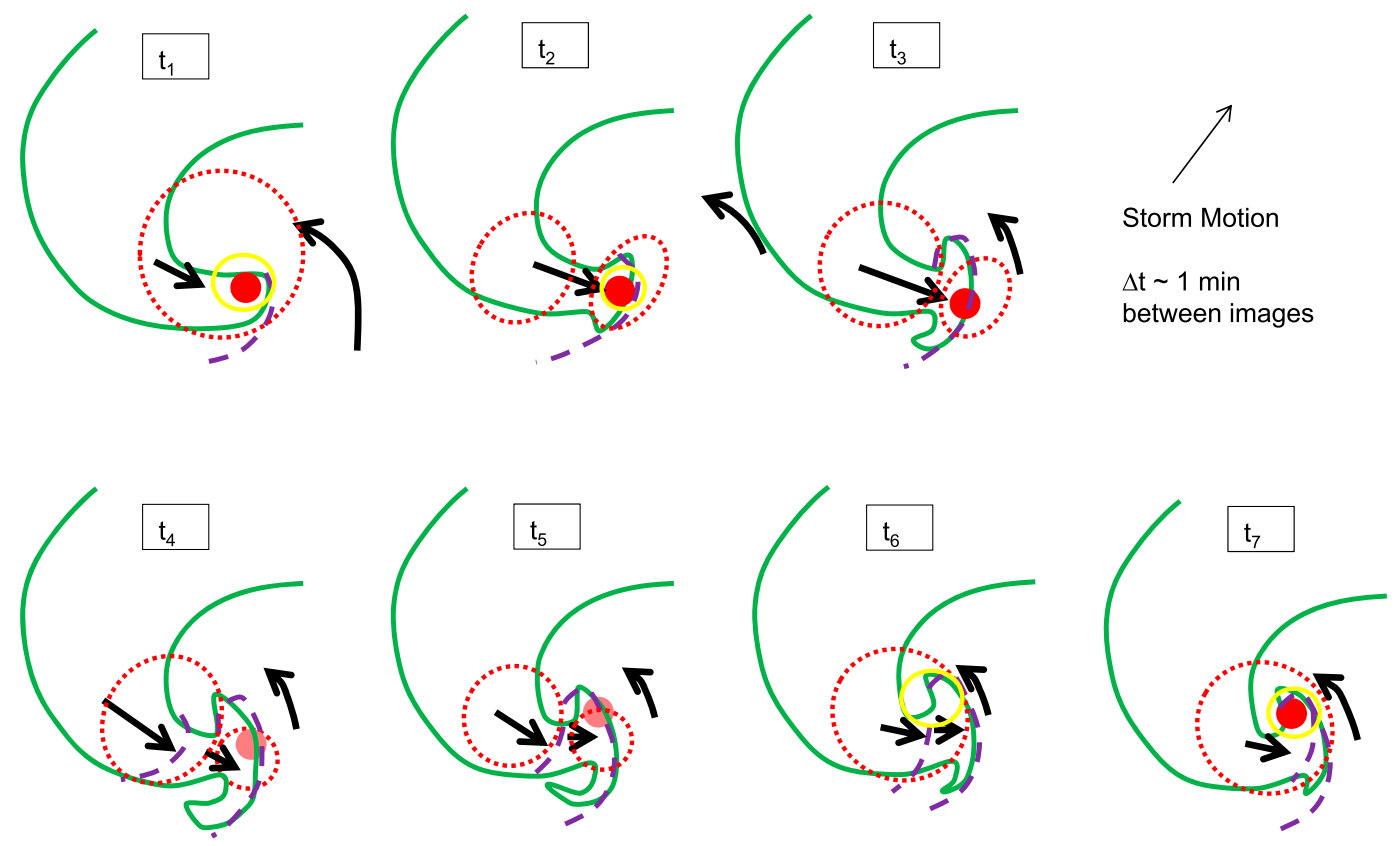

FIG. 15. Conceptual model of the evolution of low-level and midlevel features observed between the dissipation of tornado 1 and the genesis of tornado 2 . The green outline represents the 25 -dB $Z$ reflectivity contour at low levels. Red dots indicate low-level TS, pink dots indicate subtornadic circulation, yellow circles denote low-level mesocyclone prior to tornadogenesis, arrows indicate inflow/outflow, purple dashed curves indicate RFGF locations, and the red dashed ellipses indicate the locations of midlevel mesocyclones. The time between images is $\sim 1 \mathrm{~min}$.

outflow and weakening inflow $\left(t_{2}\right.$ and $\left.t_{3}\right)$. The reflectivity field takes on the appearance of a hammerhead in response to the surge in outflow behind the RFGF. The tornado decays and a secondary rear-flank momentum surge develops $\left(t_{4}\right)$. The midlevel mesocyclones remain separate, with the original one remaining larger and stronger. Subsequently, the secondary RFGF moves through the hook echo, and the remnant circulation associated with tornado 1 is advected back up the primary RFGF in response to a new developing low-level mesocyclone $\left(t_{5}\right)$. The second midlevel mesocyclone become indiscernible from the original one and the circulation broadens. Concurrently, the new low-level mesocyclone intensifies and a new subtornadic vortex develops as the secondary RFGF intersects the primary one $\left(t_{6}\right)$. Finally, the second tornado forms as the secondary RFGF occludes the new low-level mesocyclone $\left(t_{7}\right)$. This whole process occurred over a period of approximately $7 \mathrm{~min}$. Thus, if one were using data from a WSR-88D, only two volume scans would have been collected during this timeframe. It is therefore likely that the details of this evolution would have not been able to be resolved.

\section{c. Tornadogenesis}

In this case, there was no evidence of a descending TS, implying that tornadogenesis was not associated with the dynamic pipe effect in the traditional sense, reinforcing recent observations that mesocyclone-associated tornadoes may not form in this manner (Alexander 2010; F13). Rather, the appearance of a descending TVS (TS depending on resolution) may be an artifact of poor temporal sampling (F13). It appears that tornadogenesis occurred as a result of rapid convergence of air toward the axis of rotation nearly simultaneously, both aloft and at low levels. Rotation of tornadic intensity developed within one volume scan (17s) over the depth of the observations.

Around 2048 UTC rotation intensified aloft $(z \sim 3-$ $4 \mathrm{~km}$ ) but failed to do so below this layer (Fig. 12b). This intensification may have been a failed attempt at tornadogenesis from traditional DPE theory. However, the rotation did not build downward with time (unlike what would be expected according to the DPE), and strong rotation never occurred in the lowest elevation angles observed. One minute after this intensification occurred, rotation weakened again. The absence of organized lowlevel rotation is a key difference between this failed attempt and the successful tornadogenesis that occurred several minutes later. This observation lends additional support to the hypothesis that a juxtaposition of strong rotation aloft (e.g., 3-4 km) above strong near-surface rotation was required for tornadogenesis to occur for this case. 




FIG. 16. Upper-air observations from the 1800 UTC OUN (Norman, OK) rawinsonde data on 24 May 2011. LCL and LFC are indicated.

Another observation regarding the failed tornadogenesis attempt is the time increment between it and tornadogenesis, which was about $4 \mathrm{~min}$. This corresponds roughly to the time it takes for a WSR-88D to complete a volume scan. The rapid-temporal updates of RaXPol allowed these observations to be identified as distinct, separate processes. F13 performed an analysis on four rapid-scan tornadogenesis datasets from a different rapid-scanning mobile radar platform (one of which was for this case study), none of which exhibited descending TVSs in the rapid-scan data. They degraded the temporal resolution of the rapid-scan data and determined that based on the criteria Trapp et al. (1999) used to define a descending TVS all four of the cases would have been classified as having descending TVSs. Thus, with the comparatively poor temporal resolution of the WSR-88D, these two events appeared to have been associated with the same process, creating the illusion that rotation developed aloft first, and then developed near the surface. The rapid-temporal updates of RaXPol allowed these observations to be identified as distinct, separate processes.
Once the tornado formed, it quickly intensified above $\sim 1.5 \mathrm{~km}$ but more slowly below this height. It is intriguing that both the tornado decay and tornadogenesis processes appear to have a disparity between what happens above and below a layer between $\sim 1.5$ and $2 \mathrm{~km}$ AGL. According to the 1800 UTC Norman, Oklahoma, sounding (Fig. 16) there is a capping inversion between $\sim 1.5$ and $3 \mathrm{~km}$. This layer of stable air may explain why the rotation decayed here initially, and why rotation below this height intensified more slowly than that above it. ${ }^{11}$ Presumably, the rotating updraft below the inversion hit the stable layer and rising air

\footnotetext{
${ }^{11}$ The 1800 UTC sounding is likely not completely representative of the storm-scale environment owing to the temporal evolution (nearly $2 \mathrm{~h})$ and spatial separation $(\sim 100 \mathrm{~km})$ between the storm environment and the sounding location. Additionally, microphysical processes and other storm-scale processes involved with deep convection will modify the local thermodynamic environment. These considerations at least partially explain the discrepancy of the stable layer depth between the storm environment and the sounding.
} 
became negatively buoyant, relying instead upon dynamically induced vertical motions, and reducing the updraft velocity. Weak vertical velocity gradients would reduce the amount of vorticity stretching occurring, causing the tornado to intensify more slowly than in regions with higher vertical velocity gradients and, hence, greater vorticity stretching. A similar argument can be made about the decay process. Although both genesis and decay officially occurred nearly simultaneously at all heights observed, the subsequent strengthening and weakening, respectively, of the tornadic (subtornadic) velocity couplet appeared to be influenced by this stable region between 1.5 and $2 \mathrm{~km}$. F14 also observed a vertical discontinuity in TVS evolution and found that the height of the discontinuity they observed was near the height of the level of free convection.

An important caveat to this study is that the minimum beam height during tornado decay was $\sim 1 \mathrm{~km}$ AGL, and it was $\sim 650 \mathrm{~m}$ AGL during tornadogenesis. Therefore, the processes occurring below these heights remain unresolved. Despite this shortcoming, there is little reason to believe trends would be different if the lowest level data were available. The tornadogenesis process still would not display a descending tornadic vortex signature (at least not above $650-1000 \mathrm{~m}$ ), and tornado decay would still likely be similar, just with additional information about how the vortex at the lowest level was evolving. Unfortunately, the question about what occurs at these lowest levels $(z<500 \mathrm{~m})$ remains unanswered from these data.

\section{Conclusions}

This study describes the evolution of two tornadoes and the attendant storm-scale properties by examining the structure and single-Doppler kinematics of the storm using a rapid-scanning, mobile radar. The primary conclusions of this study that add to the current state of knowledge are as follows:

1) The weakening process of the first tornado occurred over a duration of $\sim 3 \mathrm{~min}$ and was nonmonotonic with time. However, when the velocity couplet weakened below the tornadic threshold, it did so within $30 \mathrm{~s}$ over the depth of the available observations (e.g., $1-4.5 \mathrm{~km}$ ).

2) The behavior of tornado dissipation is contingent upon the specification of what constitutes a tornadic $\Delta V_{\max }$. If a different $\Delta V_{\max }$ were specified in this case, the decay process (where $\Delta V_{\max }$ is reduced below the chosen tornadic threshold of $55 \mathrm{~m} \mathrm{~s}^{-1}$ ) would make it appear that the tornado first dissipated between 1.5 and $2.5 \mathrm{~km}$, then aloft, and last at the lowest levels.

3) Temporally persistent subtornadic rotation organized and intensified first at low levels $(z<1 \mathrm{~km})$. Tornadogenesis did not occur until immediately after a sudden intensification of rotation between 3 and $3.5 \mathrm{~km}$ ARL. Within $30 \mathrm{~s}$, the $\Delta V_{\max }$ increased from subtornadic to tornadic intensity $\left(>55 \mathrm{~m} \mathrm{~s}^{-1}\right)$ over all heights between $\sim 700 \mathrm{~m}$ and $3 \mathrm{~km}$ except $1.5 \mathrm{~km}$. There is no evidence of a descending TS prior to tornadogenesis.

4) Rapidly evolving storm-scale features played an important role in tornado-scale processes. These processes included an atypical occlusion or cycling process in which the tornado was advected forward, toward the RFGF, coincident with the formation of a new mesocyclone to the east-southeast of the original mesocyclone. Additionally, RFGF surges played counteracting roles in tornado evolution: the first tornado appears to have dissipated as a result of an RFGF surge, whereas the second tornado appears to have formed in part due to a secondary RFGF surge.

5) There appears to be a difference in the evolution of rotation above and below $\sim 1.5-2 \mathrm{~km}$ both during tornadogenesis and decay. This height was near the base of a strong capping inversion in the 1800 UTC KOUN sounding. Thus, the stable layer likely inhibited vertical motions in this layer. Above the inversion, parcels reached their level of free convection and could freely rise, accelerating rapidly according to the buoyancy of the air parcels.

The high temporal frequency at which observations were collected was imperative to deducing the conclusions above. Tornadogenesis and dissipation occurred over a period of less than $30 \mathrm{~s}$, and the storm-scale processes occurring just prior to these events happened on the order of $2 \mathrm{~min}$ for tornadogenesis and $4 \mathrm{~min}$ for dissipation. A period of rapid intensification transpired over the first 2 min of tornado 2's life, and some stormscale processes affecting tornadogenesis and decay (e.g., RFGF surges and cycling) occur on time scales of approximately $2-4 \mathrm{~min}$. Hence, nearly all processes examined in this study directly affecting tornado evolution would not have been resolvable with a WSR-88D, even if it were located very close to the tornado (for superior spatial resolution), owing to the slow temporal update speed of the NEXRAD system. Likewise, nonrapid scanning mobile radars would have had difficulty adequately resolving these processes' temporal evolution.

Although this study made extensive use of the radar data that were available, many questions remain unanswered. The data and analyses contribute to a more comprehensive understanding of rapidly evolving tornado 
and storm-scale processes, but the kinematics and dynamics associated with difficult-to-observe, yet very important, processes were unable to be quantified. The sources and transport of vorticity are of particular interest in this case, but they could not be determined with the data available.

Finally, this is only one case in a growing pool of studies that appear to argue against the dynamic pipe effect hypothesis of tornado formation in supercells. It would be ideal to examine many cases of varying tornado intensity and tornadogenesis failure with high temporal resolution to determine if, in fact, observations of a descending TS may indeed be an artifact of insufficient temporal sampling, as was suggested by F13.

Acknowledgments. This work was supported by NSF Grants AGS-0821231 and AGS-0934307 awarded to the second author at the University of Oklahoma. The authors additionally acknowledge the contributions of the Advanced Radar Research Center led by Robert Palmer, and particularly John Meier for hardware and software support. David Dowell provided the objective analysis code. Last, the authors acknowledge the comments and suggestions made by three anonymous reviewers who contributed to the improvement of this work and the flow of the manuscript.

\section{REFERENCES}

Adlerman, E. J., and K. K. Droegemeier, 2002: The sensitivity of numerically simulated cyclic mesocyclogenesis to variations in model physical and computational parameters. Mon. Wea. Rev., 130, 2671-2691, doi:10.1175/1520-0493(2002)130<2671: TSONSC $>2.0$.CO;2.

$\longrightarrow$, and - , 2005: The dependence of numerically simulated cyclic mesocyclogenesis upon environmental vertical wind shear. Mon. Wea. Rev., 133, 3595-3623, doi:10.1175/ MWR3039.1.

— - — , and R. Davies-Jones, 1999: A numerical simulation of cyclic mesocyclogenesis. J. Atmos. Sci., 56, 2045-2069, doi:10.1175/1520-0469(1999)056<2045:ANSOCM>2.0.CO;2.

Alexander, C. R., 2010: A mobile radar based climatology of supercell tornado structure and dynamics. Ph.D. dissertation, University of Oklahoma, Norman, Oklahoma, 229 pp.

Beck, J. R., J. L. Schroeder, and J. M. Wurman, 2006: Highresolution dual-Doppler analyses of the 29 May 2001 Kress, Texas, cyclic supercell. Mon. Wea. Rev., 134, 3125-3148, doi:10.1175/MWR3246.1.

Bluestein, H. B., and R. M. Wakimoto, 2003: Mobile radar observations of severe convective storms. Radar and Atmospheric Science: A Collection of Essays in Honor of David Atlas, Meteor. Monogr., No. 30, Amer. Meteor. Soc., 105-138, doi:10.1175/0065-9401(2003)030<0105:MROOSC>2.0.CO;2.

— W W. C. Lee, M. Bell, C. C. Weiss, and A. L. Pazmany, 2003: Mobile Doppler radar observations of a tornado in a supercell near Bassett, Nebraska, on 5 June 1999. Part II: Tornadovortex structure. Mon. Wea. Rev., 131, 2968-2984, doi:10.1175/ 1520-0493(2003)131<2968:MDROOA>2.0.CO;2.
- M. M. French, I. PopStefanija, R. T. Bluth, and J. B. Knorr, 2010: A mobile, phased-array Doppler radar for the study of severe convective storms. Bull. Amer. Meteor. Soc., 91, 579600, doi:10.1175/2009BAMS2914.1.

_ J. B. Houser, M. M. French, J. C. Snyder, G. D. Emmitt, I. PopStefanija, C. Baldi, and R. T. Bluth, 2014: Observations of the boundary layer near tornadoes and in supercells using a mobile, collocated, pulsed Doppler lidar and radar. J. Atmos. Oceanic Technol., 31, 302-325, doi:10.1175/ JTECH-D-13-00112.1.

Brandes, E. A., 1978: Mesocyclone evolution and tornadogenesis: Some observations. Mon. Wea. Rev., 106, 995-1011, doi:10.1175/1520-0493(1978)106<0995:MEATSO>2.0.CO;2.

Brooks, H. E., C. A. Doswell, and R. Davies-Jones, 1993: Environmental helicity and the maintenance and evolution of lowlevel mesocyclones. The Tornado: Its Structure, Dynamics, Prediction, and Hazards, Geophys. Monogr., Vol. 79, Amer. Geophys. Union, 97-104.

Brown, R. A., and V. T. Wood, 2012: The tornadic vortex signature: An update. Wea. Forecasting, 27, 525-530, doi:10.1175/ WAF-D-11-00111.1.

— L. R. Lemon, and D. W. Burgess, 1978: Tornado detection by pulsed Doppler radar. Mon. Wea. Rev., 106, 29-38, doi:10.1175/1520-0493(1978)106<0029:TDBPDR>2.0.CO;2.

Burgess, D. W., L. R. Lemon, and R. A. Brown, 1975: Tornado characteristics revealed by Doppler radar. Geophys. Res. Lett., 2, 183-184, doi:10.1029/GL002i005p00183.

_, V. T. Wood, and R. A. Brown, 1982: Mesocyclone evolution statistics. Preprints, 12th Conf. on Severe Local Storms, San Antonio, TX, Amer. Meteor. Soc., 422-424.

Byko, Z., P. Markowski, Y. Richardson, J. Wurman, and E. Adlerman, 2009: Descending reflectivity cores in supercell thunderstorms observed by mobile radars and in a highresolution numerical simulation. Wea. Forecasting, 24, 155186, doi:10.1175/2008WAF2222116.1.

Davies-Jones, R. P., 2015: A review of supercell and tornado dynamics. Atmos. Res., 158-159, 274-291, doi:10.1016/ j.atmosres.2014.04.007.

— , R. J. Trapp, and H. B. Bluestein, 2001: Tornadoes and tornadic storms. Severe Convective Storms, Meteor. Monogr., No. 28, Amer. Meteor. Soc., 167-222.

Doviak, R. J., and D. Zrnić, 2006: Doppler Radar and Weather Observations. 2nd ed. Dover Publications, 562 pp.

Dowell, D. C., and H. B. Bluestein, 1997: The Arcadia, Oklahoma, storm of 17 May 1981: Analysis of a supercell during tornadogenesis. Mon. Wea. Rev., 125, 2562-2582, doi:10.1175/ 1520-0493(1997)125<2562:TAOSOM>2.0.CO;2.

— , and - 2002a: The 8 June 1995 McLean, Texas, storm. Part I: Observations of cyclic tornadogenesis. Mon. Wea. Rev., 130, 2626-2648, doi:10.1175/1520-0493(2002)130<2626: TJMTSP $>2.0 . \mathrm{CO} ; 2$.

— , and — 2 2002b: The 8 June 1995 McLean, Texas, storm. Part II: Cyclic tornado formation, maintenance, and dissipation. Mon. Wea. Rev., 130, 2649-2670, doi:10.1175/1520-0493(2002)130<2649: TJMTSP $>2.0 . \mathrm{CO} ; 2$.

Dunn, L. B., and S. V. Vasiloff, 2001: Tornadogenesis and operational considerations of the 11 August 1999 Salt Lake City tornado as seen from two different Doppler radars. Wea. Forecasting, 16, 377-398, doi:10.1175/1520-0434(2001)016<0377: TAOCOT $>2.0 . \mathrm{CO} ; 2$.

French, M. M., H. B. Bluestein, D. C. Dowell, L. J. Wicker, M. R. Kramar, and A. L. Pazmany, 2008: High-resolution, mobile Doppler radar observations of cyclic mesocyclogenesis in a 
supercell. Mon. Wea. Rev., 136, 4997-5016, doi:10.1175/ 2008MWR2407.1.

,-- I. Popstefanija, C. A. Baldi, and R. T. Bluth, 2013: Reexamining the vertical development of tornadic vortex signatures in supercells. Mon. Wea. Rev., 141, 4576-4601, doi:10.1175/MWR-D-12-00315.1.

$-, \ldots, \ldots, \ldots$, and $\_,$2014: Mobile, phased-array, Doppler radar observations of tornadoes at X-band. Mon. Wea. Rev., 142, 1010-1036, doi:10.1175/MWR-D-13-00101.1.

Kosiba, K. A., and J. Wurman, 2013: The three-dimensional structure and evolution of a tornado boundary layer. Wea. Forecasting, 28, 1552-1561, doi:10.1175/WAF-D-13-00070.1.

- - - Y. Richardson, P. Markowski, P. Robinson, and J. Marquis, 2013: Genesis of the Goshen County, Wyoming, tornado on 5 June 2009 during VORTEX2. Mon. Wea. Rev., 141, 1157-1181, doi:10.1175/MWR-D-12-00056.1.

Kumjian, M. R., and A. V. Ryzhkov, 2008: Polarimetric signatures in supercell thunderstorms. J. Appl. Meteor. Climatol., 47, 1940-1961, doi:10.1175/2007JAMC1874.1.

,-- , V. M. Melnikov, and T. J. Schuur, 2010: Rapid-scan super-resolution observations of a cyclic supercell with a dualpolarization WSR-88D. Mon. Wea. Rev., 138, 3762-3786, doi:10.1175/2010MWR3322.1.

Lee, B. D., C. A. Finley, and T. M. Samaras, 2011: Surface analysis near and within the Tipton, Kansas, tornado on 29 May 2008. Mon. Wea. Rev., 139, 370-386, doi:10.1175/2010MWR3454.1.

—_ - - and C. D. Karstens, 2012: The Bowdle, South Dakota, cyclic tornadic supercell of 22 May 2010: Surface analysis of rearflank downdraft evolution and multiple internal surges. Mon. Wea. Rev., 140, 3419-3441, doi:10.1175/MWR-D-11-00351.1.

Lemon, L. R., and C. A. Doswell, 1979: Severe thunderstorm evolution and mesocyclone structure as related to tornadogenesis. Mon. Wea. Rev., 107, 1184-1197, doi:10.1175/ 1520-0493(1979)107<1184:STEAMS >2.0.CO;2.

Leslie, L. M., 1971: The development of concentrated vortices: A numerical study. J. Fluid Mech., 48, 1-21, doi:10.1017/ S0022112071001435.

Markowski, P. M., and Y. P. Richardson, 2009: Tornadogenesis: Our current understanding, forecasting considerations, and questions to guide future research. Atmos. Res., 93, 3-10, doi:10.1016/j.atmosres.2008.09.015.

Marquis, J., Y. Richardson, P. Markowski, D. Dowell, and J. Wurman, 2012: Tornado maintenance investigated with high-resolution dual-Doppler and EnKF analysis. Mon. Wea. Rev., 140, 3-27, doi:10.1175/MWR-D-11-00025.1.

NWS, 2011a: The Lookeba tornado of May 24, 2011. Accessed 27 April 2015. [Available online at http://www.srh.noaa.gov/oun/ ?n=events-20110524-tornado-b1.]

_ 2011b: The Calumet-El Reno-Piedmont-Guthrie tornado of May 24, 2011. Accessed 27 April 2015. [Available online at http://www.srh.noaa.gov/oun/?n=events-20110524-tornado-b2.]

_ 2011c: Information about the May 24, 2011 tornado outbreak: Public information statement about tornado damage. Accessed 27 April 2015. [Available online at http://www.srh. noaa.gov/oun/?n=events-20110524-tornado-b2.]

Palmer, R. D., and Coauthors, 2011: Observations of the 10 May 2010 tornado outbreak using OU-PRIME: Potential for new science with high-resolution polarimetric radar. Bull. Amer. Meteor. Soc., 92, 871-891, doi:10.1175/2011BAMS3125.1.

Pazmany, A. L., J. B. Mead, H. B. Bluestein, J. C. Snyder, and J. B. Houser, 2013: A mobile rapid-scanning X-band polarimetric (RaXPol) Doppler radar system. J. Atmos. Oceanic Technol., 30, 1398-1413, doi:10.1175/JTECH-D-12-00166.1.
Rasmussen, E. N., J. M. Straka, M. S. Gilmore, and R. DaviesJones, 2006: A preliminary survey of rear-flank descending reflectivity cores in supercell storms. Wea. Forecasting, 21, 923-938, doi:10.1175/WAF962.1.

Roberts, R. D., and J. W. Wilson, 1995: The genesis of three nonsupercell tornadoes observed with dual-Doppler radar. Mon. Wea. Rev., 123, 3408-3436, doi:10.1175/1520-0493(1995)123<3408: TGOTNT $>2.0 . \mathrm{CO} ; 2$.

Skinner, P. S., C. C. Weiss, M. M. French, H. B. Bluestein, P. M. Markowski, and Y. P. Richardson, 2014: VORTEX2 observations of a low-level mesocyclone with multiple internal rearflank downdraft momentum surges in the 18 May 2010, Dumas, Texas, supercell. Mon. Wea. Rev., 142, 2935-2960, doi:10.1175/MWR-D-13-00240.1.

Smith, R. K., and L. M. Leslie, 1979: A numerical study of tornadogenesis in a rotating thunderstorm. Quart. J. Roy. Meteor. Soc., 105, 107-127, doi:10.1002/qj.49710544308.

Snyder, J. C., and H. B. Bluestein, 2014: Some considerations for the use of high-resolution mobile radar data in tornado intensity determination. Wea. Forecasting, 29, 799-827, doi:10.1175/WAF-D-14-00026.1.

,,-- G. Zhang, and S. J. Frasier, 2010: Attenuation correction and hydrometeor classification of high-resolution, $\mathrm{X}$-band, dual-polarized mobile radar measurements in severe convective storms. J. Atmos. Oceanic Technol., 27, 1979-2001, doi:10.1175/2010JTECHA1356.1.

,,-- V. Venkatesh, and S. J. Frasier, 2013: Observations of polarimetric signatures in supercells by an X-band mobile Doppler radar. Mon. Wea. Rev., 141, 3-29, doi:10.1175/ MWR-D-12-00068.1.

Tanamachi, R. L., H. B. Bluestein, J. B. Houser, S. J. Frasier, and K. M. Hardwick, 2012: Mobile, X-band, polarimetric Doppler radar observations of the 4 May 2007 Greensburg, Kansas, tornadic supercell. Mon. Wea. Rev., 140, 2103-2125, doi:10.1175/MWR-D-11-00142.1.

Trapp, R. J., and B. H. Fiedler, 1995: Tornado-like vortexgenesis in a simplified numerical model. J. Atmos. Sci., 52, 3757-3778, doi:10.1175/1520-0469(1995)052<3757:TLVIAS>2.0.CO;2.

- and R. Davies-Jones, 1997: Tornadogenesis with and without a dynamic pipe effect. J. Atmos. Sci., 54, 113-133, doi:10.1175/1520-0469(1997)054<0113:TWAWAD>2.0.CO;2.

—, E. D. Mitchell, G. A. Tipton, D. W. Effertz, A. I. Watson, D. L. Andra, and M. A. Magsig, 1999: Descending and nondescending tornadic vortex signatures detected by WSR-88Ds. Wea. Forecasting, 14, 625-639, doi:10.1175/1520-0434(1999)014<0625: DANTVS $>2.0 . \mathrm{CO} ; 2$.

Van Den Broeke, M. S., J. M. Straka, and E. N. Rasmussen, 2008: Polarimetric radar observations at low levels during tornado life cycles in a small sample of classic southern plains supercells. J. Appl. Meteor. Climatol., 47, 1232-1247, doi:10.1175/ 2007JAMC1714.1.

Vasiloff, S. V., 1993: Single-Doppler radar study of a variety of tornado types. The Tornado: Its Structure, Dynamics, Prediction, and Hazards, Geophys. Monogr., Vol. 79, Amer. Geophys. Union, 105-114.

Wakimoto, R. M., and N. T. Atkins, 1996: Observations on the origins of rotation: The Newcastle tornado during VORTEX 94. Mon. Wea. Rev., 124, 384-407, doi:10.1175/1520-0493(1996)124<0384: OOTOOR $>2.0 . \mathrm{CO} ; 2$.

— , and C. Liu, 1998: The Garden City, Kansas, storm during VORTEX 95. Part II: The wall cloud and tornado. Mon. Wea. Rev., 126, 393-408, doi:10.1175/1520-0493(1998)126<0393: TGCKSD $>2.0 . C O ; 2$. 
- H. V. Murphy, and H. Cai, 2004: The San Angelo, Texas, supercell of 31 May 1995: Visual observations and tornadogenesis. Mon. Wea. Rev., 132, 1269-1293, doi:10.1175/ 1520-0493(2004)132<1269:TSATSO > 2.0.CO;2.

— N N. T. Atkins, and J. Wurman, 2011: The LaGrange tornado during VORTEX2. Part I: Photogrammetric analysis of the tornado combined with single-Doppler radar data. Mon. Wea. Rev., 139, 2233-2258, doi:10.1175/2010MWR3568.1.

Wicker, L. J., and R. B. Wilhelmson, 1995: Simulation and analysis of tornado development and decay within a three-dimensional supercell thunderstorm. J. Atmos. Sci., 52, 2675-2703, doi:10.1175/1520-0469(1995)052<2675:SAAOTD>2.0.CO;2.

Wurman, J., 2002: The multiple-vortex structure of a tornado. Wea. Forecasting, 17, 473-505, doi:10.1175/1520-0434(2002)017<0473: TMVSOA $>2.0 . \mathrm{CO} ; 2$.

-, and M. Randall, 2001: An inexpensive, mobile, rapid-scan radar. Preprints, 30th Conf. on Radar Meteorology, Munich, Germany, Amer. Meteor. Soc., 98-100.
_ , and K. Kosiba, 2013: Finescale radar observations of tornado and mesocyclone structures. Wea. Forecasting, 28, 1157-1174, doi:10.1175/WAF-D-12-00127.1.

— , Y. P. Richardson, C. Alexander, S. Weygandt, and P. F. Zhang, 2007: Dual-Doppler analysis of winds and vorticity budget terms near a tornado. Mon. Wea. Rev., 135, 2392-2405, doi:10.1175/MWR3404.1.

—, K. Kosiba, P. Markowski, Y. Richardson, D. Dowell, and P. Robinson, 2010: Finescale single- and dual-Doppler analysis of tornado intensification, maintenance, and dissipation in the Orleans, Nebraska, supercell. Mon. Wea. Rev., 138, 44394455, doi:10.1175/2010MWR3330.1.

Ziegler, C. L., E. N. Rasmussen, T. R. Shepherd, A. I. Watson, and J. M. Straka, 2001: The evolution of low-level rotation in the 29 May 1994 Newcastle-Graham, Texas, storm complex during VORTEX. Mon. Wea. Rev., 129, 1339-1368, doi:10.1175/1520-0493(2001)129<1339: TEOLLR > 2.0.CO;2. 